\title{
Uncertainty and Growth Disasters
}

Jovanovic, Boyan and Sai Ma

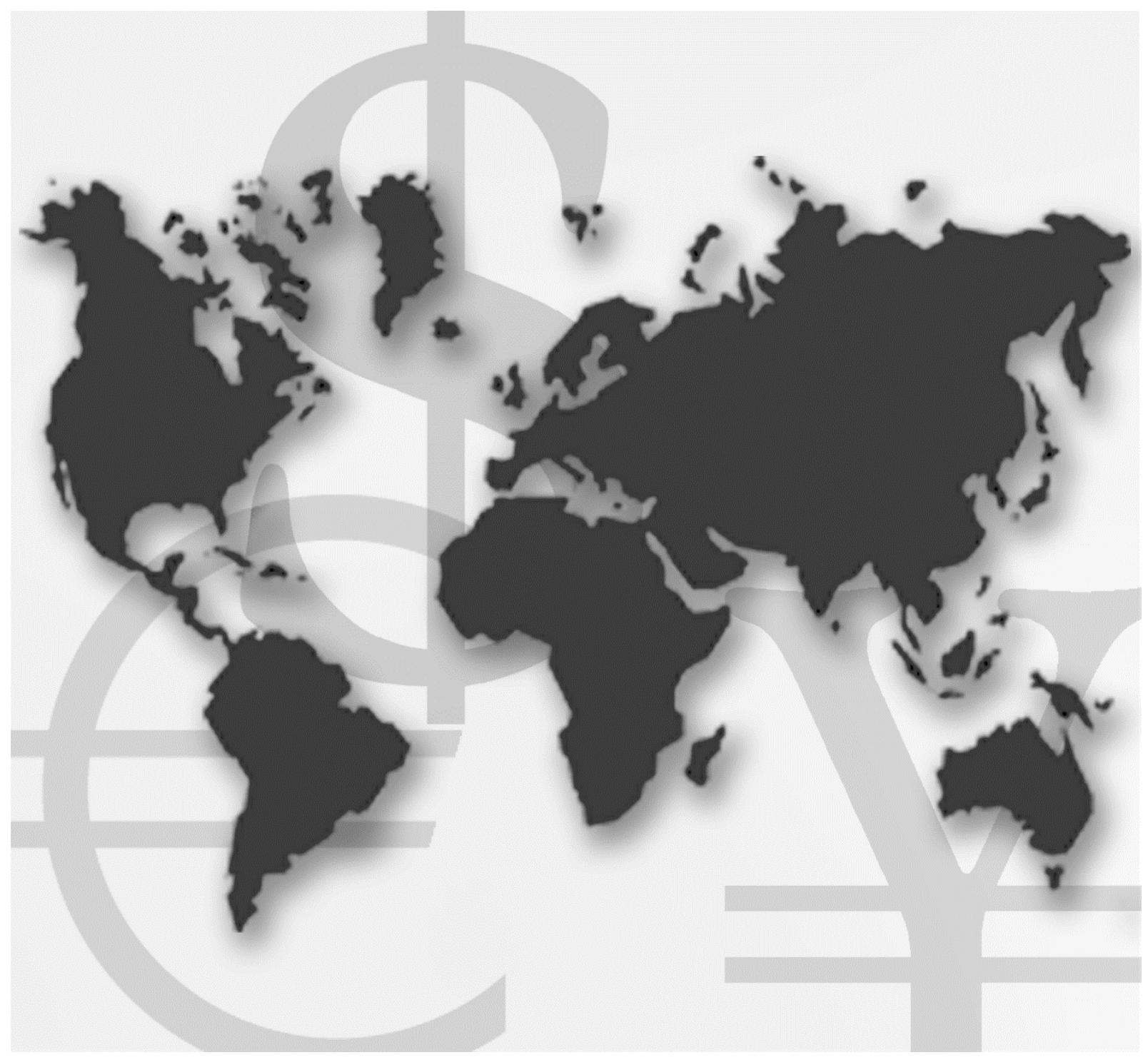

\section{International Finance Discussion Papers}

Board of Governors of the Federal Reserve System

Number 1279

May 2020 


\section{Board of Governors of the Federal Reserve System \\ International Finance Discussion Papers}

Number 1279

May 2020

\section{Uncertainty and Growth Disasters}

Boyan Jovanovic and Sai Ma

NOTE: International Finance Discussion Papers (IFDPs) are preliminary materials circulated to stimulate discussion and critical comment. The analysis and conclusions set forth are those of the authors and do not indicate concurrence by other members of the research staff or the Board of Governors. References in publications to the International Finance Discussion Papers Series (other than acknowledgement) should be cleared with the author(s) to protect the tentative character of these papers. Recent IFDPs are available on the Web at www.federalreserve.gov/pubs/ifdp/. This paper can be downloaded without charge from the Social Science Research Network electronic library at www.ssrn.com. 


\title{
Uncertainty and Growth Disasters
}

\author{
BOYAN JOVANOVIC \\ SAI MA* \\ New York University \\ Federal Reserve Board
}

March 2020

\begin{abstract}
This paper documents several stylized facts on the real effects of economic uncertainty. First, higher uncertainty is associated with a more dispersed and negatively skewed distribution of output growth. Second, the response of economic growth to an increase in uncertainty is highly nonlinear and asymmetric. Third, higher asset volatility magnifies the negative impact of uncertainty on growth. We develop and estimate an analytically tractable model in which rapid adoption of new technology may raise economic uncertainty which causes measured productivity to decline. The equilibrium growth distribution is negatively skewed and higher uncertainty leads to a thicker left tail.
\end{abstract}

JEL Code: D80, E44, O40, O47

Keywords: Uncertainty and growth, volatility, downside risk, growth at risk

*Jovanovic is with Department of Economics, New York University (boyan.jovanovic@nyu.edu). Ma is with the Board of Governors of the Federal Reserve System, Division of International Finance (Corresponding author). C. Ave \& 20th Street NW, Washington, DC 20551. Email: sai.ma@frb.gov. The views expressed are those of the authors and not necessarily those of the Federal Reserve Board or the Federal Reserve System. All errors are ours. First draft: May 2019. 


\section{Introduction}

Contemporary macro literature often finds uncertainty about the future to be an important driver of economic fluctuations. A growing body of work proposes uncertainty as a cause of economic slowdown and sluggish recovery. For example, Bloom (2009) and Bloom, Floetotto, Jaimovich, Saporta-Eksten and Terry (2018) argue that higher uncertainty stems from the process governing technological innovation, which subsequently causes a decline in real activity.

Empirically, this evidence has been found to be robust to the use of various proxy variables such as implied stock volatility (VIX), economic policy uncertainty (EPU) from Baker, Bloom and Davis (2016), or a broad-based measure of macroeconomic and financial uncertainty, as in Jurado, Ludvigson and Ng (2015) (JLN) and Ludvigson, Ma and Ng (2019) (LMN). However, these papers usually investigate the impact of higher uncertainty on expected mean growth either via a linear forecasting regression or a structural vector autoregression (SVAR). Consequently, they are silent about the effect of uncertainty on the volatility or other higher moments of the growth distribution, and this may underestimate the impact of economic uncertainty on growth downside risk.

In this paper, we provide evidence that uncertainty is highly correlated with the higher moments of the growth distribution. Figure 1 depicts the contemporaneous relationship between JLN macro uncertainty and 36-month rolling window average industrial production (IP) growth (left panel), growth volatility (middle panel), and growth skewness (right panel). While uncertainty is highly negatively correlated $(-36 \%)$ with the mean growth as shown in the literature, we find that uncertainty is also highly correlated with growth volatility (44\%) and growth skewness (-22\%), respectively. Therefore, higher uncertainty is not only associated with lower mean growth but also contributes to a more disperse and negatively skewed growth distribution.

We empirically estimate the distribution of future real growth in industrial production as a function of uncertainty measures using quantile regression methods. ${ }^{1}$ We document three stylized facts from the estimations. First, higher economic uncertainty is associated with a more dispersed and left-skewed future growth distribution. A one-standarddeviation increase in uncertainty statistically significantly increases the interquartile range of the one-month ahead annualized growth distribution by $2 \%$, and decreases the lower 5 th percentile by $5 \%$. This indicates that the marginal effects of higher uncertainty are

\footnotetext{
${ }^{1}$ This empirical model is based on Adrian, Boyarchenko and Giannone (2019) (ABG). Instead of using uncertainty measure, $\mathrm{ABG}$ uses national financial condition index (NFCI) as the conditional variable to estimate the growth distribution.
} 

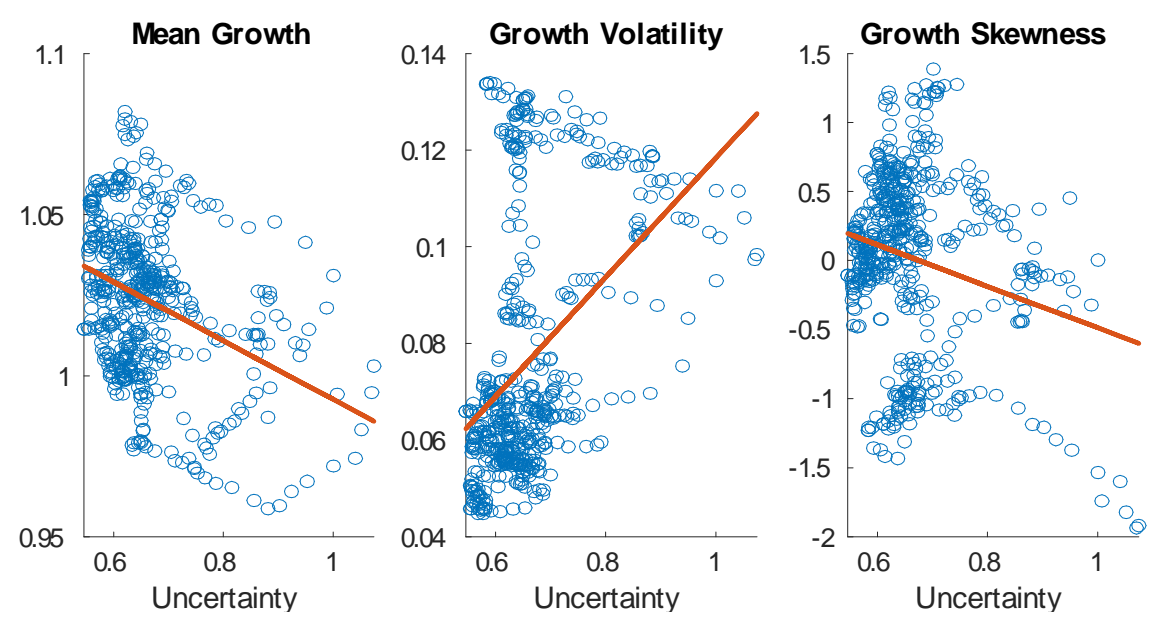

Figure 1: Uncertainty and Higher Moments of Growth. Note: this figure depicts the contemporaneous relationship between JLN macro uncertainty and 36-month rolling window average IP growth (left panel), growth volatility (middle panel), and growth skewness (right panel). The sample spans the period 1971:01 to 2018:12.

to significantly increase growth downside risk.

Second, the response of IP growth to changes in uncertainty is highly asymmetric, the response being much higher when uncertainty rises than when it falls. An increase in uncertainty is associated with a larger decrease in the lower tail of the growth distribution while it has a much smaller impact on its upper tail. These results suggest that higher uncertainty could lead to an abrupt economic decline whereas lower uncertainty does not necessarily rebound the economy from the recession. Third, higher asset volatility magnifies the negative impact of uncertainty on growth. We find that when the asset market is more volatile as measured by higher VIX, an increase in macro uncertainty has a larger negative impact on the lower tail of the expected growth distribution. A one standard deviation increase in VIX increases in the marginal effect of uncertainty on the lower 5 th percentile by $10 \%$.

Motivated by this empirical evidence, we present and estimate an analytically tractable model that generate endogenous growth and uncertainty. The equilibrium growth is the result of the adoption of technologies of uncertain quality. A technology's "quality" refers to how closely the technology's needs match the economy's input endowments. Since technology needs are unpredictable, rapid irreversible adoption of new technologies can lead to high uncertainty and decline in the growth.

In equilibrium, uncertainty affects growth because firms adopt technologies the exact character of which they do not know. How well a technology fits a firm's asset endowments is revealed only after the fact. The mismatch is distributed symmetrically but the cost of 
that mismatch is quadratic so that maximal losses from technology adoption exceed the maximal gains. The firm's growth rate is therefore negatively skewed as observed in the data.

The model provides an analytic characterization of the equilibrium growth distribution. Consistent with our empirical evidence, higher uncertainty about the newly adopted technologies leads to a lower average growth as well as a more dispersed and negatively skewed growth distribution. By assuming that the uncertainty has no long-run impacts on growth, the calibrated model is able to quantitatively match the marginal effect of uncertainty on several key moments of the growth distribution. As in the data, the model suggests that higher asset price volatility, measured from the estimated option prices of a Lucas (1978) representative security, leads to a higher marginal effect of uncertainty on the lower tail of the growth distribution.

\section{Related Literature}

Our paper relates closely to two major strands of literature. First, a growing body of empirical studies the real effect of uncertainty. Carriero, Clark and Marcellino (2018) finds that economic uncertainty has a strong negative effect on economic outcomes. Similarly, based on breaks in the volatility of macroeconomic variables, Angelini, Bacchiocchi, Caggiano and Fanelli (2019) shows that macro uncertainty has a contractionary effect on output. Using a shock-restricted SVAR approach, Ludvigson et al. (2019) finds that financial uncertainty could be a possible source of business cycle fluctuation. In spite of the mixed evidence on which type of uncertainty has a contractionary effect on economic growth, it's evidential that the higher uncertainty about future economy exhibits large impact on expected mean growth. However, all these papers empirically investigate uncertainty and real variables using structural vector autoregression (SVAR) framework and thus cannot establish the relationship between uncertainty and higher moments of the growth distribution. ${ }^{2}$

One exception is Hengge (2019). As in our paper, she uses quantile regression analysis and shows that the relationship between macroeconomic uncertainty and future quarterly GDP growth is highly nonlinear and asymmetric. Our empirical evidence on the asymmetric response of expected IP growth to higher uncertainty is consistent with hers despite using monthly industrial production as the measure of economic growth. Using quantile regression estimates, we further construct the Growth-at-Risk measure and study

\footnotetext{
${ }^{2}$ Other examples include Caldara, Fuentes-Albero, Gilchrist and Zakrajšek (2016), Alfaro, Bloom and Lin (2016), and Shin and Zhong (2018).
} 
its interaction with asset pricing volatility and capacity utilization both empirically and theoretically. To the best of our knowledge, ours is the first paper to provide a theoretical framework to study the impact of uncertainty on higher moments of the growth distribution.

Second, a large body of theoretical literature proposes that higher economic uncertainty as a cause of the decline in growth. This includes models of the real options effects of uncertainty (Bernanke (1983), McDonald and Siegel (1986)), models in which uncertainty influences financing constraints (Gilchrist, Sim and Zakrajsek (2010), Arellano, Bai and Kehoe (2011)), investment (Fajgelbaum, Schaal and Taschereau-Dumouchel (2017)), or precautionary saving (Basu and Bundick (2017), Leduc and Liu (2016), FernándezVillaverde, Pablo Guerrón-Quintana and Uribe (2011)). Our notion of uncertainty in the model is similar to Bloom (2009) and Bloom et al. (2018) that assumes that higher uncertainty originates directly in the process governing technological innovation and adoption.

Our model follows the putty-clay tradition of Johansen (1959), assuming irreversible technological commitment. It builds on Jovanovic (2006) by deriving the closed-form expression of equilibrium growth, and studying the relationship between uncertainty and higher moments of the growth distribution. ${ }^{3}$

The rest of this paper is organized as follows. Section 2 provides some empirical evidence on the asymmetric real effect of uncertainty. Section 3 presents the model, analyzes the model's empirical implications, and provides an extended model with learning. Section 4 concludes the paper.

\section{Empirical Evidence}

In this section, we describe the data and present the key empirical fact that the relationship between future economic growth and uncertainty is highly nonlinear. To document this feature in the data, we follow Adrian et al. (2019) and investigate the relationship between uncertainty and future growth via forecasting quantile regressions. Compared to the traditional OLS forecasting regressions, quantile regressions describe how the set of conditional variables, including uncertainty measures, affect different quantiles of the future growth. This methodology thus allows the estimated relationship between uncertainty and future growth to differ across quantiles. This extension of a simple linear regression model can capture the potential nonlinear relationship between the shocks in uncertainty and vulnerability of the growth, which is largely neglected in the literature.

\footnotetext{
${ }^{3}$ Other related models are Ramey and Ramey (1991), Chalkley and Lee (1998), Veldkamp (2005), Klenow (1998), and Acemoglu and Scott (1997).
} 
More formally, we regress the $h$-month-ahead real industrial production growth (thereafter "IP growth") on a vector of condition variables $\mathbf{x}_{t}$,

$$
\Delta i p_{t+h, \alpha}=\boldsymbol{\delta}_{\alpha, h}^{\prime} \mathbf{x}_{t}+\varepsilon_{t}
$$

where the conditional variables $\mathbf{x}_{t}$ include a constant, the lag of the IP growth, and uncertainty $U_{t}$.

The regression slope can be solved by minimizing the quantile-weighted absolute value of errors:

$$
\hat{\boldsymbol{\delta}}_{\alpha, h}=\arg \min \sum_{t}\left\{\begin{array}{c}
\alpha \mathbf{1}_{\Delta i p_{t+h, \alpha}>\boldsymbol{\delta}_{\alpha, h}^{\prime} \mathbf{x}_{t}}\left|\Delta i p_{t+h, \alpha}-\boldsymbol{\delta}_{\alpha}^{\prime} \mathbf{x}_{t}\right| \\
+(1-\alpha) \mathbf{1}_{\Delta i p_{t+h, \alpha}<\boldsymbol{\delta}_{\alpha, h}^{\prime} \mathbf{x}_{t}}\left|\Delta i p_{t+h, \alpha}-\boldsymbol{\delta}_{\alpha}^{\prime} \mathbf{x}_{t}\right|
\end{array}\right\}
$$

where $h$ is the forecasting horizon, $\alpha$ is the quantile and $\mathbf{1}$ is the indicator variable. For inference, standard errors are estimated via the bootstrap.

\subsection{Data}

The monthly industrial production data is obtained from FRED Economic database maintained by the Federal Reserve Bank of St. Louis. Our main measures of uncertainty $U_{t}$ are constructed following the framework of Jurado et al. (2015) and Ludvigson et al. (2019), which aggregates over a large number of estimated uncertainties constructed from a panel of data. Let $y_{j t}^{C} \in Y_{t}^{C}=\left(y_{1 t}^{C}, \ldots, y_{N_{C} t}^{C}\right)^{\prime}$ be a variable in category $C$. Its $h$-period ahead uncertainty, denoted by $\mathcal{U}_{j t}^{C}(h)$, is defined to be the volatility of the purely unforecastable component of the future value of the series, conditional on all information available. Specifically,

$$
\mathcal{U}_{j t}^{C}(h) \equiv \sqrt{\mathbb{E}\left[\left(y_{j t+h}^{C}-\mathbb{E}\left[y_{j t+h}^{C} \mid I_{t}\right]\right)^{2} \mid I_{t}\right]}
$$

where $I_{t}$ denotes the information available. Uncertainty in category $C$ is an aggregate of individual uncertainty series in the category:

$$
U_{C t}(h) \equiv \operatorname{plim}_{N_{C} \rightarrow \infty} \sum_{j=1}^{N_{C}} \frac{1}{N_{C}} \mathcal{U}_{j t}^{C}(h) \equiv \mathbb{E}_{C}\left[\mathcal{U}_{j t}^{C}(h)\right]
$$

If the expectation today of the squared error in forecasting $y_{j t+h}$ rises, uncertainty in the variable increases. In this paper, we focus on macro uncertainty and use a monthly macro dataset, consisting of 134 mostly macroeconomic time series taken from McCracken and 

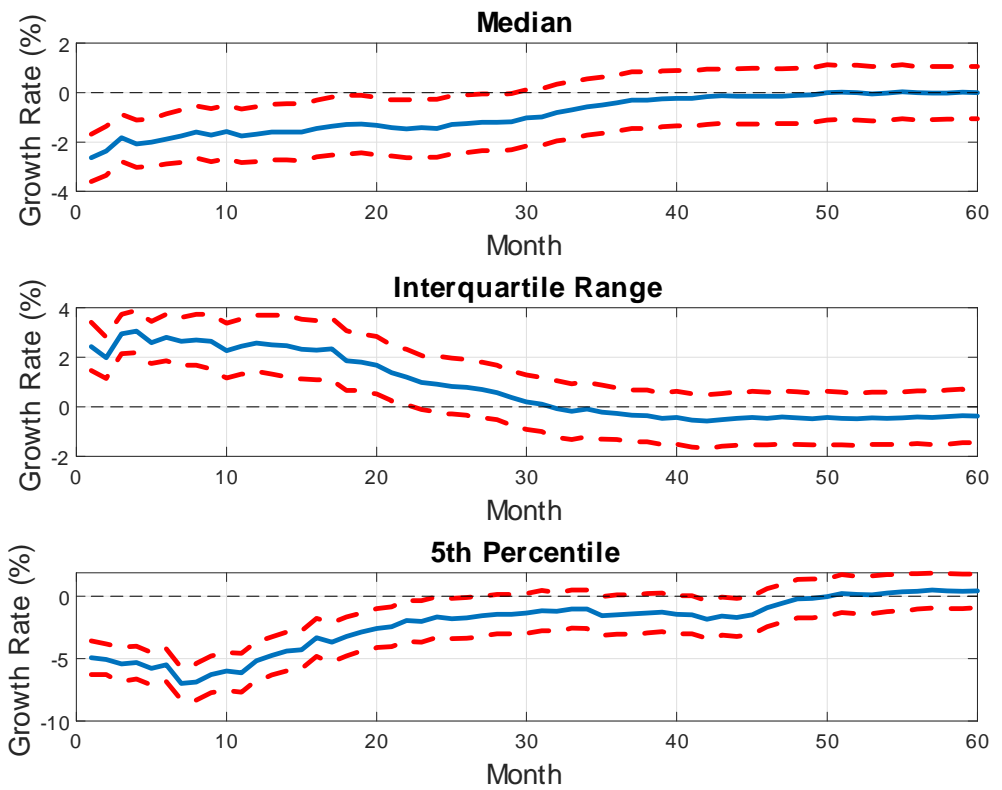

Figure 2: Impact of a one-standard deviation increase in uncertainty measure on the annualized growth rate. Note: this figure report the estimated coefficient of uncertainty over $h=$ $1,2 \ldots, 60$ months from the baseline quantile regression described in the texts. The bootstrapped $67 \%$ confidence intervals are reported in red dashed lines.

$\mathrm{Ng}$ (2016). ${ }^{4}$ Following Ludvigson et al. (2019), 1-month ahead macro uncertainty as our baseline measure of uncertainty. Our sample is monthly and spans the period 1971:01 to 2018:12, unless otherwise noted.

\subsection{Results}

By construction, any effects of changes in $\mathbf{x}_{t}$ on $h$-month ahead growth at $\alpha$ percentile is captured by the slope estimate $\hat{\boldsymbol{\delta}}_{\alpha, h}$. Figure 2 reports the estimates of marginal coefficient of uncertainty over $h=1,2 \ldots, 60$ months. The top panel reports the effect of uncertainty on changes in expected median growth. We find that a one-standard-deviation increase in uncertainty decreases the median of the annualized growth by $3 \%$ over the next month. The effect gradually weakens over time with a half-life of 23 months. The middle panel shows that the distribution of the growth becomes more dispersed following an increase in uncertainty. The interquartile range rises at short- and medium- terms. Similarly, the bottom panel shows that when uncertainty increases, the distribution of the growth is more negatively skewed. The 5th percentile of the growth falls by $6 \%$ at short horizon,

\footnotetext{
${ }^{4}$ Therefore, the category $C$ is set to be the macro category $M$ in Ludvigson et al. (2019).
} 


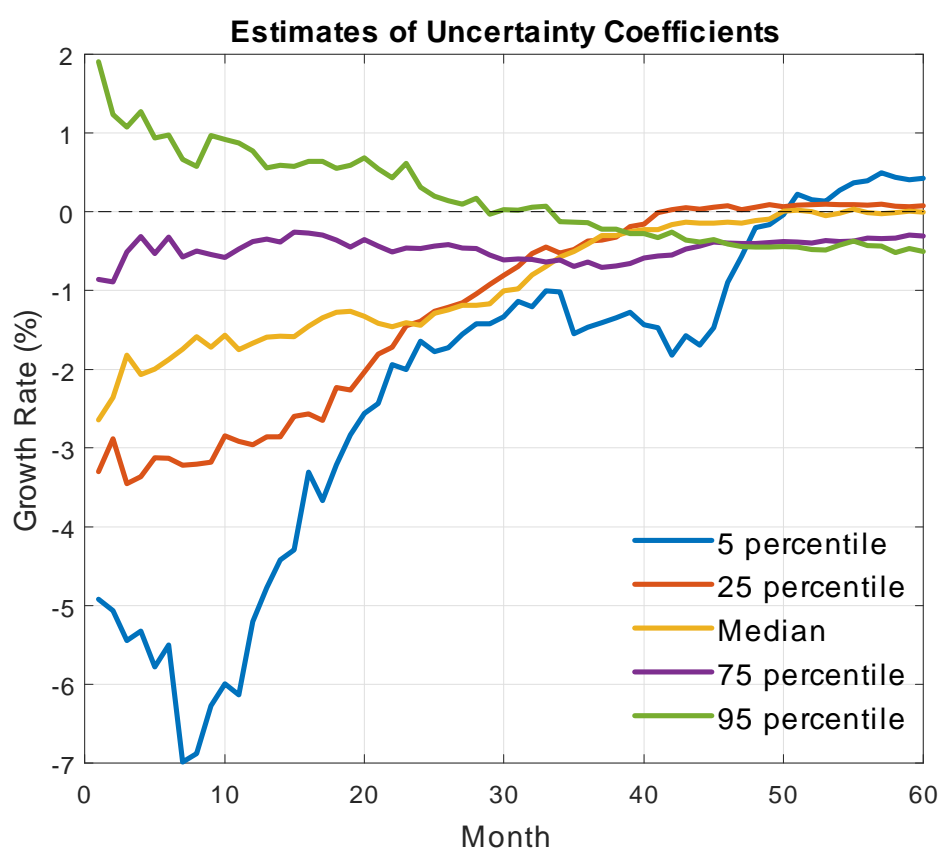

Figure 3: Asymmetric impact of uncertainty on growth. Note: this figure reports the estimated uncertianty coefficients for different quantiles $\alpha$ over horizon $h$ from the baseline quantile regression described in the texts.

and $2 \%$ over 2 to 4 years. As a result, the impact of the uncertainty on skewness of the distribution is long-lasting.

In Figure 3, we plot the estimated uncertainty coefficients $\boldsymbol{\delta}_{\alpha, h}$ for different $\alpha$ over horizon $h$. In most cases, an increase in uncertainty is associated with a larger decrease in the lower tail of the growth distribution while it has much smaller impact on the upper tail. This asymmetric response shows that the impact of uncertainty on growth is highly nonlinear.

\subsection{Growth-at-Risk}

The Growth-at-Risk (GaR) in our paper is defined as the estimated lower 5th percentile of the distribution of expected real IP growth. It's worth noting that our measure of GaR is different from the one in Adrian, Grinberg, Liang and Malik (2018) because they use the national financial condition index (NFCI) from the Chicago Fed in conditional variables $x_{t}$ whereas we include the uncertainty measure. ${ }^{5}$ Figure 4 shows the time series of onemonth ahead GaR estimated using uncertainty measure. The red line in the figure shows

\footnotetext{
${ }^{5}$ In addition, Adrian et al. (2018) uses quarterly GDP growth whereas we use monthly industrial production as the measure of economic growth.
} 
the he GaR estimated using NFCI for comparisons. First, it shows that both measures of GaR are counter-cyclical and with a less than $5 \%$ probability, a one-standard deviation increase in uncertainty is associated with as large as $3 \%$ decline in IP Growth within a month during recessions. Second, GaR estimated with uncertainty is more volatile and exhibit larger spikes during recessions. For the rest of the paper, we refer to GaR as the one that is estimated using uncertainty.

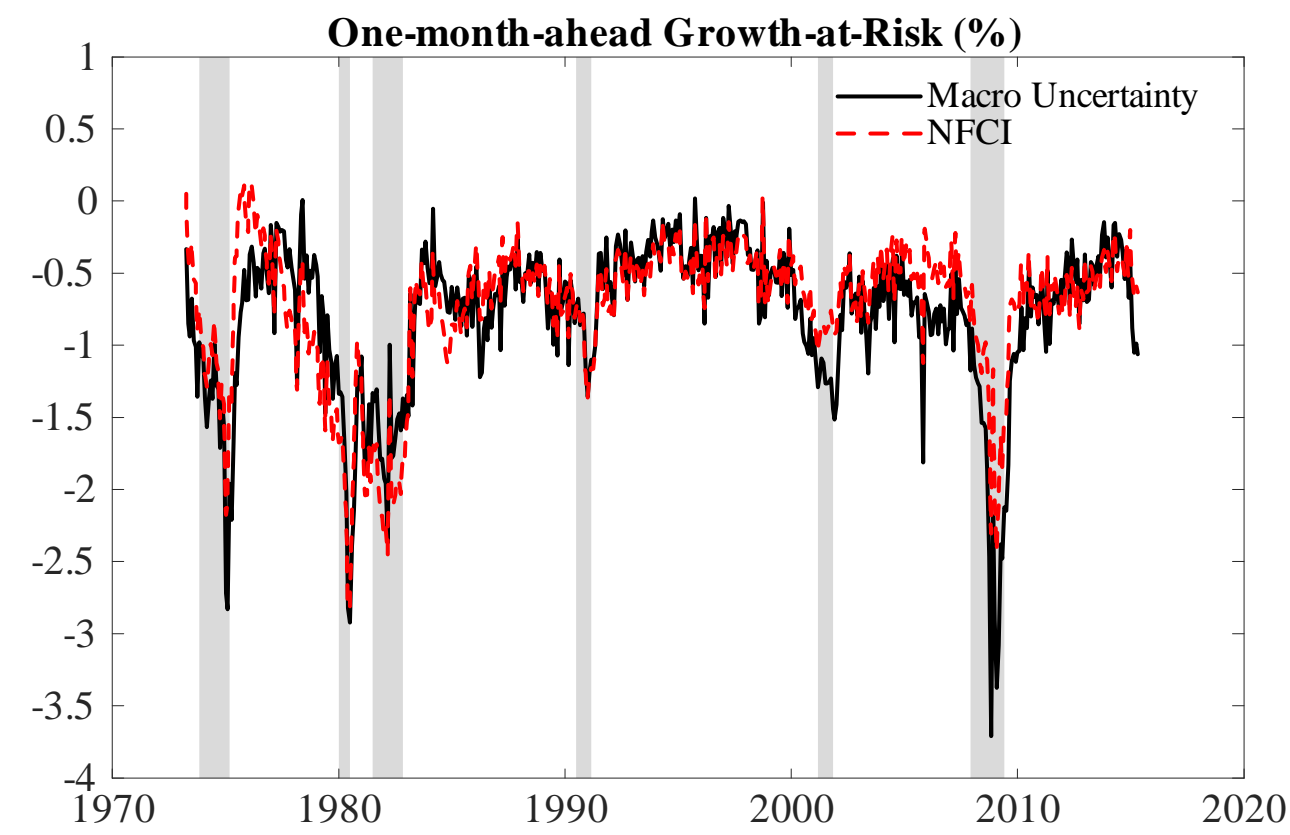

Figure 4: Growth-at-Risk. Note: this figure reports the estimated Growth-at-Risk using NFCI (red line) and JLN Macro uncertainty (black line). The baseline quantile regression is described in the texts. The sample spans the period 1971:01 to 2018:12.

Our baseline measure of uncertainty is based on macro variables but some papers in the literature, such as Ludvigson et al. (2019), found evidence that volatility related to the asset returns also lead to persistent decline IP growth. This evidence suggests that the uncertainty should be associated with a larger decline in growth when the asset prices are more volatile. To directly test this argument, Figure 4 plots the estimated GaR against the standardized implied volatility indices (VIX), which is a popular measure of the stock market's expectation of volatility implied by S\&P 500 index options. ${ }^{6}$ It shows that when asset market is more volatile, an increase in macro uncertainty has a larger negative impact on IP growth and thus leads to a lower GaR. On average, a two-standard-deviation

\footnotetext{
${ }^{6}$ In the paper, we chose to use the VXO implied volatility index that is available since 1962 instead of VIX because the latter is only available after 1990 and the two measures are $99 \%$ correlated.
} 
increase in VIX is associated with a $0.5 \%$ decline in GaR.

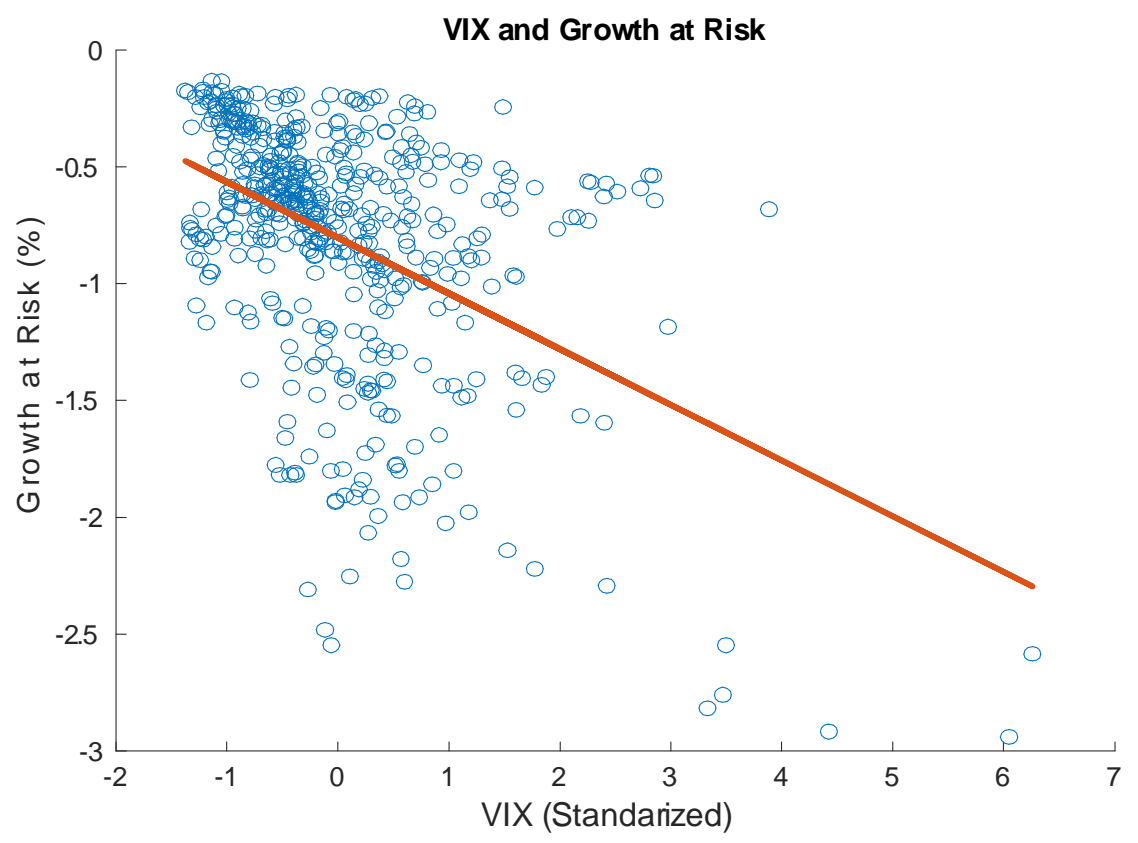

Figure 5: Growth-at-Risk and VIX. Note: this figure shows the contempraneous relationship between standardized VIX and estimated Growth-at-Risk. The Growth-at-Risk is estimated conditional on JLN macro uncertainty. The red line reports the OLS Estimate. The sample spans the period 1971:01 to $2018: 12$.

To sum up, we document some empirical evidence on the real effect of uncertainty. First, we find that an increase in uncertainty leads to lower median and yet more volatile IP growth, and a more negative GaR. Second, the response of IP growth to an increase in uncertainty is highly nonlinear, especially at the short term. Third, higher asset volatility magnifies the negative effect of uncertainty on growth. In the next section, we set up a tractable model that is able to capture these key features in the data.

\section{Model}

The model features a single agent "Crusoe" , his preferences over consumption sequences $\left(c_{t}\right)$ are

$$
E_{0}\left\{\sum_{0}^{\infty} \beta^{t} \ln c_{t}\right\} .
$$

Production - For simplicity of the exposition, we assume there is a single production good $y$ and there is no physical capital. The output depends on the level of technology $A$ 
as well as Crusoe's skill mix $h$. The potential output, $y^{p}$, is defined as

$$
y^{p}=\exp \left\{A-\frac{\lambda}{2}\left(s_{A}-h^{\prime}\right)^{2}\right\} \text {. }
$$

where $s_{A}$ is the skill-mix ideal for technology $A$. By construction, $\frac{\lambda}{2}\left(s_{A}-h^{\prime}\right)^{2}$ captures the foregone output due to the "skill gap" between Crusoe's current skill and the ideal skill for technology $A$.

Adoption of Technology - Crusoe can raise his technology by any amount, $x \geq 0$, so that starting today at $A$, tomorrow's technology is

$$
A^{\prime}=A+x .
$$

Crusoe must use technology $A^{\prime}$ for at least one period. We assume that adopting a new technology is free but adoption of the new technology $A^{\prime}$ makes unpredictable demands on the skill mix,

$$
s_{A^{\prime}}=s_{A}+x \varepsilon
$$

where $\varepsilon \sim F(\varepsilon)$ is time specific and i.i.d., having mean zero and variance $\sigma^{2}$. Once $\varepsilon$ is drawn, $s_{A^{\prime}}$ becomes an invariant skill requirement for technology $A^{\prime}$. Crusoe chooses $A^{\prime}$ before seeing $\varepsilon$, and he cannot return to technologies that he used in the past.

Adjustment of $h$.- Crusoe starts the period with $h$. Before producing, he can change it to $h^{\prime} \equiv h+\Delta$ at a adjustment cost of

$$
C\left(y^{p}, \Delta\right) \equiv\left[1-\exp \left\{-\frac{\theta}{2} \Delta^{2}\right\}\right] y^{p} .
$$

Finally, Crusoe's net output is

$$
y^{p}-C\left(y^{p}, \Delta\right) \equiv y(u, \Delta, A)=\exp \left\{A-\frac{\lambda}{2}(u-\Delta)^{2}-\frac{\theta}{2} \Delta^{2}\right\},
$$

where

$$
u=s_{A}-h,
$$

is the gap between ideal and actual skill (hereafter the "skill gap").

\subsection{Optimal Skill Investment}

The state consists of the technology level and his skill gap $(u, A)$, and his decisions are $(x, \Delta)$. He has no assets other than $h$ and $A$, and he simply consumes his output. His Bellman equation is

$$
V(u, A)=\max _{x, \Delta}\left\{\ln y(u, \Delta, A)+\beta \int V(u+x \varepsilon-\Delta, A+x) d F(\varepsilon)\right\} .
$$

The solution to (5), derived in Appendix 1, can be summarized as follows: 
Proposition 1 (Jovanovic 2006) The policy functions are

$$
x=\frac{1}{\theta \sigma^{2}(1-\beta)(1-\alpha)},
$$

and

$$
\Delta=(1-\alpha) u,
$$

where

$$
\alpha=\frac{1}{2 \beta}\left\{1+\beta+\frac{\lambda}{\theta}-\sqrt{\left(1+\beta+\frac{\lambda}{\theta}\right)^{2}-4 \beta}\right\},
$$

is the fraction of the gap that Crusoe leaves open. The solution for $V$ is

$$
V(u, A)=\frac{A}{1-\beta}-\frac{1}{2} \theta(1-\alpha) u^{2}+\Upsilon,
$$

where

$$
\Upsilon=\frac{\beta}{1-\beta}\left(\frac{x}{1-\beta}-\left[\frac{\psi}{1-\beta \alpha^{2}}\right] x^{2} \sigma^{2}\right), \text { and } \psi=\frac{1}{2}\left(\lambda \alpha^{2}+\theta(1-\alpha)^{2}\right) .
$$

Proposition 1 states that Crusoe's adjustment of skills $\Delta$ is proportional to his current skill gap $u$. As a result, using equation (3) and (4), the skill gap $u$ follows a AR(1) process as follows,

$$
u_{t+1}=\alpha u_{t}+x \varepsilon_{t+1} .
$$

Uncertainty - Our notion of uncertainty is captured by the variance of the skill gap $u$. Because $\alpha$ is between zero and one, $u_{t}$ is stationary and its variance is

$$
\tau \equiv \operatorname{Var}(u)=\frac{x^{2} \sigma^{2}}{1-\alpha^{2}}=\left((1-\beta)^{2}\left(1-\alpha^{2}\right) \theta^{2}(1-\alpha)^{2}\right)^{-1} \sigma^{2},
$$

where the second equality comes from equation (6). Conditional on the current skill gap $u$, an increase in Crusoe's skill investment, through lower $\alpha$ or $\theta$, leads to lower uncertainty. In addition, noisier $\varepsilon$ (higher $\sigma^{2}$ ) leads to higher uncertainty.

Free-riding incentives and decentralization of the optimum -If all agents start in the same state and then take the same action, the equilibrium is symmetric and it has only aggregate risk. Section 4 of Jovanovic (2006) analyzes the incentives of agents to deviate from this situation by considering the option to "wait and see" how other firms fare with the newly adopted technology. The decentralized version assumes that there are two markets: A market for output, and a market for firm's shares - the only assets available to households. No markets exist for either $A$ which, since it was free for Crusoe to augment, naturally would also be freely copied in a decentralized setting, and yet firms 
still reject the option to wait and see. ${ }^{7}$ On the other hand, a firm cannot buy $h$ from other firms; one interpretation of $h$ is that of organization capital in the sense of Prescott and Visscher (1980).

\subsection{Growth Distribution}

Suppose that $C\left(y^{p}, \Delta\right)$ consists entirely of foregone output. The log of measured output then is,

$$
\begin{aligned}
\ln y_{t} & =A-\frac{\lambda}{2} \alpha^{2} u_{t}^{2}-\frac{\theta}{2}(1-\alpha)^{2} u_{t}^{2} \\
& =A_{0}+x t-\psi u_{t}^{2}
\end{aligned}
$$

Because $u_{t}$ is stationary, $\ln y$ is trend-stationary, the trend and the long-run rate of output growth is $x$. The log growth can be expressed as,

$$
g_{t+1} \equiv \ln y_{t+1}-\ln y_{t}=x-\psi\left(u_{t+1}^{2}-u_{t}^{2}\right) .
$$

Equation (13) shows that the growth is driven not just by the adoption of new technology, $x$, as studied in the traditional macro literature. It is also by the changes in Crusoe's skill gap $u_{t+1}^{2}-u_{t}^{2}$. On one hand, a decrease in the skill gap stimulates the growth. On the other hand, adjusting $h$ towards its technologically ideal value is costly and will therefore take time, but output will fall sharply whenever an unlucky draw of $s_{A}$ occurs. So we should expect the growth distribution is negative skewed especially when $\theta$ is large. This leads to the following proposition.

Proposition 2 Condition on initial output and technology level $y_{0}$ and $A_{0}$, if $\varepsilon_{t}$ follows a normal distribution $N\left(0, \sigma^{2}\right)$, the distribution of growth $g_{t}$ is left skewed and is expressed as

$$
g_{t}=x-\psi\left[C_{t}+\left(1-\alpha^{2}\right) \tau \xi_{t}^{2}+\left(\alpha A_{t}-B_{t}\right) \alpha^{t} u_{0} \xi_{t}\right]
$$

\footnotetext{
${ }^{7}$ One could add features that would imply a coexistence of heterogeneous technologies in a puttyclay framework. These include a labor market (Johansen (1959)) and firm-specific shocks to the cost of investment (Campbell (1998) and Gilchrist and Williams (2000)). These features would allow the model to match facts about technology-diffusion lags.
} 
where $\xi_{t} \sim N(0,1)$ follows standard normal distribution and

$$
\begin{aligned}
A_{t} & =\sqrt{4\left(1-\alpha^{2}\right) \tau\left(\frac{1-\alpha^{2 t}}{1-\alpha^{2}}+1\right)} \\
B_{t} & =\sqrt{4\left(1-\alpha^{2}\right) \tau\left(\frac{1-\alpha^{2 t}}{1-\alpha^{2}}\right)} \\
C_{t} & =\left(\alpha^{t} u_{0}\right)^{2}-\left(\alpha^{t-1} u_{0}\right)^{2} \\
u_{0} & =\sqrt{\frac{1}{\psi}\left(\ln y_{0}-A_{0}\right)^{2}} .
\end{aligned}
$$

Because $\xi_{t}$ follows a normal distribution, $\xi_{t}^{2}$ follows the chi-squared distribution with the degree of freedom of one. Since $\psi>0$, the distribution of $\Delta \ln y_{t}$ is negatively skewed. The following corollary characterizes the first two moments of the growth distribution, such mean, median, variance and interquartile range (IQR).

Corollary 1 In equilibrium, the distribution of growth $g_{t+1}$ satisfies

$$
\begin{aligned}
E\left(g_{t+1}\right) & =x-\psi C_{t+1}-\psi\left(1-\alpha^{2}\right) \tau \\
\text { Median }\left(g_{t+1}\right) & =x-\psi C_{t+1}-0.47 \psi\left(1-\alpha^{2}\right) \tau \\
\mathbb{V}\left(g_{t+1}\right) & =2 \psi^{2}\left(1-\alpha^{2}\right)^{2} \tau^{2}+\psi^{2}\left(\left(\alpha A_{t+1}-B_{t+1}\right) \alpha^{t} u_{0}\right)^{2} \\
\operatorname{IQR}\left(g_{t+1}\right) & =\psi\left(1.22\left(1-\alpha^{2}\right) \tau+1.34\left(\alpha A_{t}-B_{t}\right) \alpha^{t} u_{0}\right) .
\end{aligned}
$$

Mean and median of the growth distribution are decreasing in uncertainty $\tau$ whereas the variance and $I Q R$ increases in uncertainty,

$$
\frac{\partial E\left(g_{t+1}\right)}{\partial \tau}<0, \frac{\partial M e d i a n\left(g_{t+1}\right)}{\partial \tau}<0, \frac{\partial \mathbb{V}\left(g_{t+1}\right)}{\partial \tau}>0, \frac{\partial I Q R\left(g_{t+1}\right)}{\partial \tau}>0 .
$$

As uncertainty increases, the average and median growth decreases while the growth distribution becomes more dispersed. In the long run, we find that the growth distribution doesn't depend on the initial values of $u_{0}$ and measures of centrality and dispersion of the growth distribution are bounded as long as $u_{t}$ is stationary. The following corollary summarizes this finding.

Corollary 2 When $t \rightarrow \infty$, the (long run) distribution of growth $g_{t}$ doesn't depend on the initial condition $u_{0}$ and satisfies

$$
g_{t}=x-\psi x^{2} \sigma^{2} \xi_{t}^{2}
$$


The first two moments of the growth distribution are bounded in the limit and satisfy the following expressions if and only if $\alpha<1$,

$$
\begin{aligned}
\lim _{t \rightarrow \infty} E(g) & =x-\psi\left(1-\alpha^{2}\right) \tau \\
\lim _{t \rightarrow \infty} \mathbb{V}(g) & =2 \psi^{2}\left(1-\alpha^{2}\right)^{2} \tau^{2} \\
\lim _{t \rightarrow \infty} \operatorname{Median}(g) & =x-0.47 \psi\left(1-\alpha^{2}\right) \tau \\
\lim _{t \rightarrow \infty} \operatorname{IQR}(g) & =1.22 \psi\left(1-\alpha^{2}\right) \tau .
\end{aligned}
$$

Growth-at-Risk (GaR) - Following Adrian et al. (2018), the Growth-at-Risk (GaR) at time $t$ is defined as the lower 5 th percentile of the growth distribution $g_{t+1}$. Using the fact that 95th percentile of the standard normal is 1.65 and the 95th percentile of chisquare is 3.84, GaR $\chi_{t}$ in our model can be expressed as

$$
\chi_{t}=x-\psi C_{t+1}-3.84 \psi\left(1-\alpha^{2}\right) \tau+1.65 \psi\left(\alpha A_{t+1}-B_{t+1}\right) \alpha^{t} u_{0}
$$

In equilibrium, GaR decreases in uncertainty $(\tau)$,

$$
\frac{\partial \chi_{t}}{\partial \tau}<0
$$

Consistent with the empirical finding, larger uncertainty is associated with higher downside risk.

\subsection{Impact of Uncertainty on Growth}

We now examine the impact of uncertainty on growth. Starting with $\sigma=1$, we raise the $\sigma$ to 1.05 and 1.15 so that $\tau$, the variance of $u$, increases by $15 \%$ and $30 \%$, respectively. A $15 \%$ increase in uncertainty corresponds to a one-standard-deviation increase in the empirical measure of uncertainty.

In order to capture the transitory impact of uncertainty as in the empirical section, we further restrict parameters so that the uncertainty shock does not have a permanent effect on the distribution of the growth rate. More specifically, we adjust the parameter pair $(\lambda, \theta)$ so that the long-run mean and variance of the growth rate do not change with their long-run values remaining at

$$
\begin{aligned}
\lim _{t \rightarrow \infty} E\left(g_{t+1}\right) & =x-\psi x^{2} \sigma^{2}=1.5 \%, \text { and } \\
\lim _{t \rightarrow \infty} V\left(g_{t+1}\right) & =2 \psi^{2} x^{4} \sigma^{4}=1 \% .
\end{aligned}
$$


We simulate the model for 60 periods and compute the changes in growth relative to the case that $\sigma^{2}$ is fixed at 1 . Figure 6 depicts the response of median growth in the top panel , interquartile range in the middle panel and 5 th percentile $(\mathrm{GaR})$ in the bottom panel to a unexpected increase in uncertainty at time 0 . The blue line depicts the case with small uncertainty $(\sigma=1.05)$ while the red line reports results with large uncertainty $(\sigma=1.15)$. The dotted black line depicts the data counterpart reported in Figure 2. Similar to the empirical evidence, an increase in uncertainty is associated with a lower median growth, higher interquartile range, and lower Growth-at-Risk. Quantitatively, our model suggests that one standard deviation increase in uncertainty immediately results in $2 \%$ decline in median growth, $3 \%$ increase in the growth dispersion and a $6 \%$ decline in GaR. Compared to the data, our model fits the impact response well but underpredicts (overpredicts) the median and GaR (interquartile range) in the medium term. This suggests the effect of uncertainty is more persistent in the data.
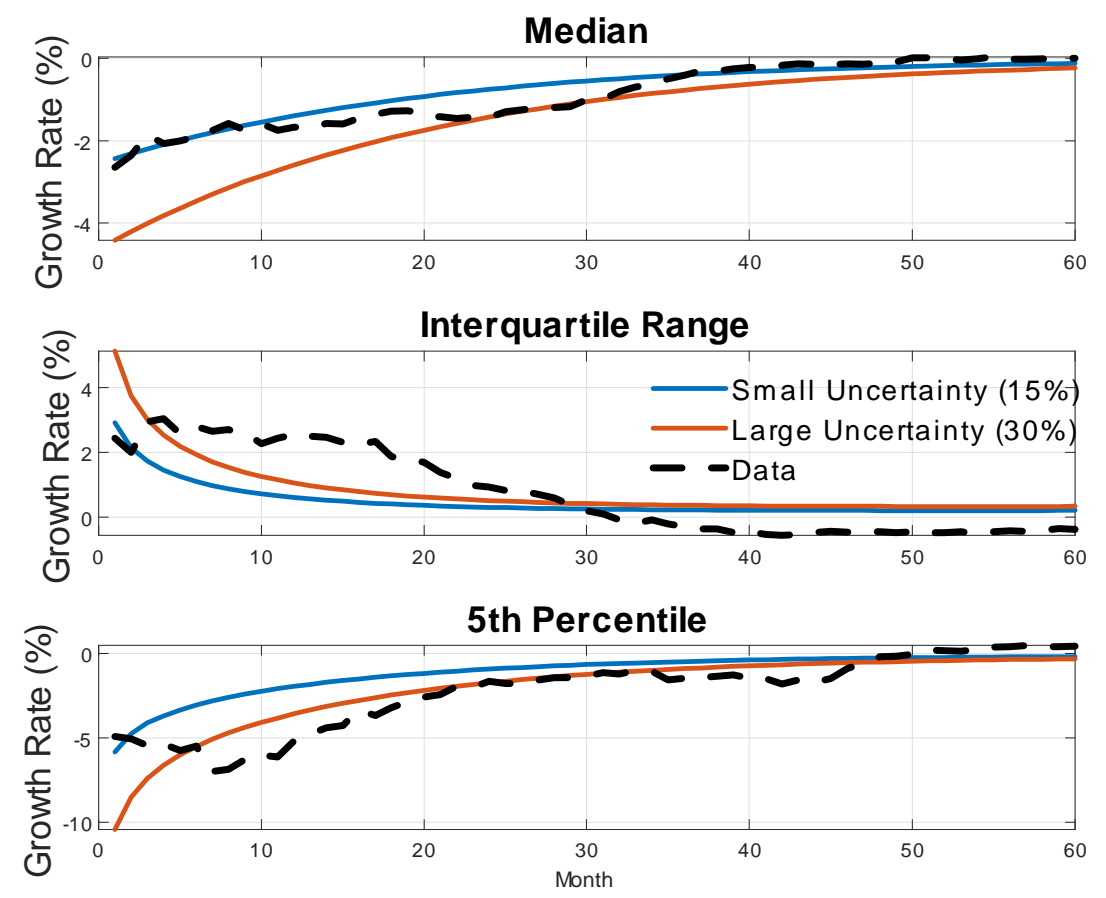

Figure 6: Uncertainty and Growth. Note: this figure depicts the response of median growth in the top panel, interquartile range in the middle panel and 5th percentile $(\mathrm{GaR})$ in the bottom panel to an unexpected $15 \%$ (blue line) and $30 \%$ increase (red line) in uncertainty at time 0 . The dashed black line is the empirical estimates from the baseline quantile regression described in the texts.

Figure 7 depicts the response of various quantiles of the expected growth distribution to 

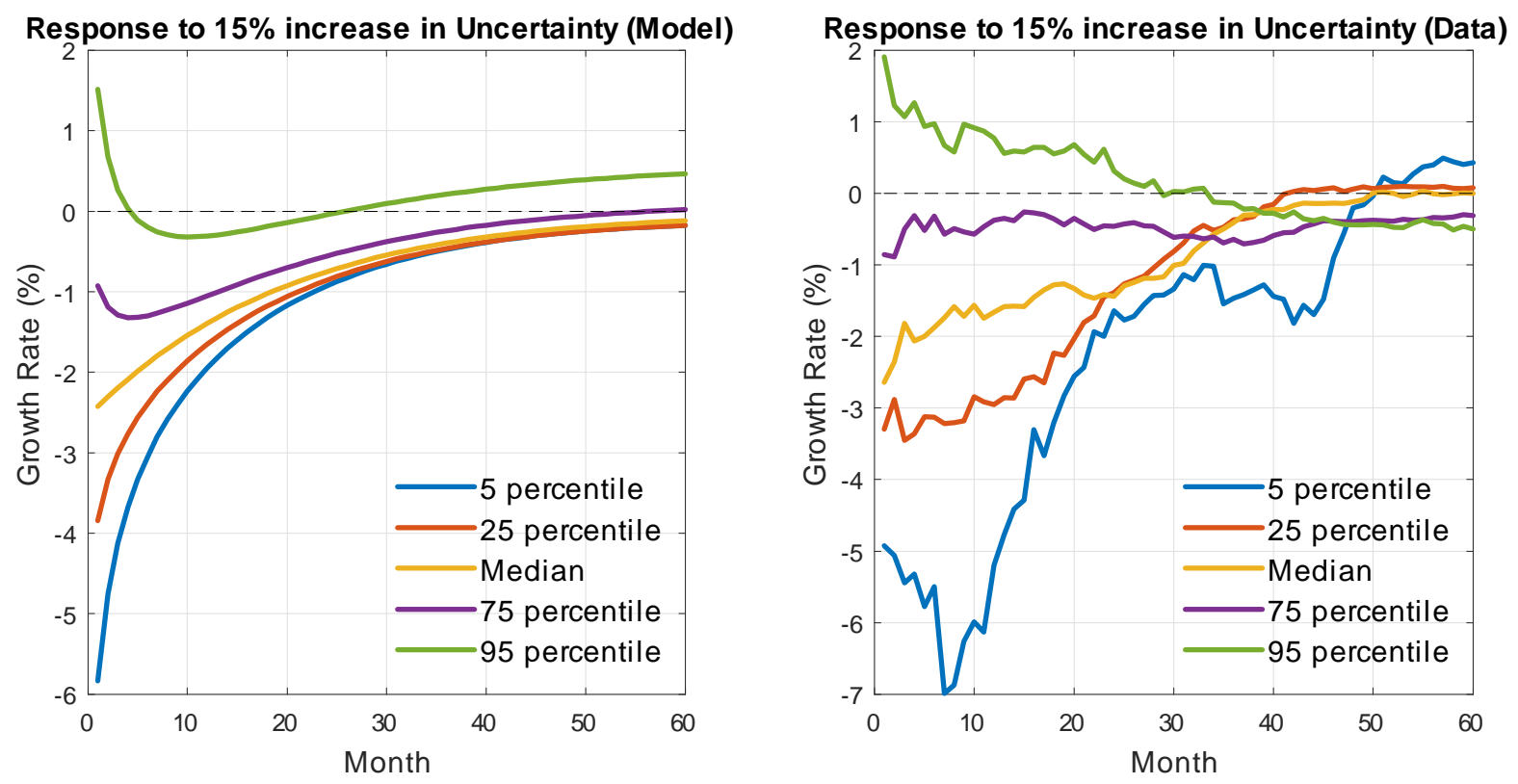

Figure 7: Asymmetric impact of uncertainty on growth. Note: the left panel depicts the response of various quantiles of the expected growth distribution to a $15 \%$ increase in uncertainty. The right panel reports the the empirical estimates from the baseline quantile regression described in the texts.

a $15 \%$ increase in uncertainty $(\sigma=1.05)$. Consistent with the empirical evidence reported in Figure 3, the response of the expected growth is highly nonlinear: an increase in uncertainty is associated with a larger decrease in the lower tail of the growth distribution whereas it has much smaller impact on the upper tail. Quantitatively, a $15 \%$ increase in uncertainty results in $4 \%$ decrease in 25 th percentile of the expected growth distribution but only contributes to a $1 \%$ decline of the 75 th percentile.

\subsection{Growth-at-Risk, the value of options and VIX}

For the price of a representative security $p(u, A)$ we have the Lucas (1978) equation

$$
p(u, A)=\beta \int \frac{c(u, A)}{c\left(u^{\prime}, A+x\right)}\left[c\left(u^{\prime}, A+x\right)+p\left(u^{\prime}, A+x\right)\right] d \Phi,
$$

because $\frac{U^{\prime}\left(c\left(u^{\prime}, A+x\right)\right)}{U^{\prime}(c(u, A))}=\frac{c(u, A)}{c\left(u^{\prime}, A+x\right)}$. Although preferences being log, prices will fluctuate because consumption growth is autocorrelated. If low consumption today means high consumption growth, a disaster is accompanied by very low asset prices. A put option ${ }^{8}$ is

\footnotetext{
${ }^{8}$ From Investopedia: A put option is an option contract giving the owner the right, but not the obligation, to sell a specified amount of an underlying security at a specified price within a specified time frame.
} 
more valuable in such states, i.e., more likely to be "in the money." The opposite for call options. Therefore today's prices of those two derivative assets should be a good signal of Growth-at-Risk. Taking today's price as the strike price for both assets,

$$
\begin{aligned}
p^{\text {put }} & =\int_{p\left(u^{\prime}, A+x\right) \leq p(u, A)}\left(p(u, A)-p\left(u^{\prime}, A+x\right)\right) d \Phi \\
p^{\text {call }} & =\int_{p\left(u^{\prime}, A+x\right) \geq p(u, A)}\left(p\left(u^{\prime}, A+x\right)-p(u, A)\right) d \Phi .
\end{aligned}
$$

The VIX index used in the empirical exercise contains put and call options on the individual firms in the S\&P 500 index, and its price would reflect idiosyncratic as well as aggregate risk. The natural definition in our model is the sum of the prices of a call option and a put option with a strike price tomorrow equal to today's price of the security $p(u, A)$,

$$
\operatorname{VIX}(u, A) \equiv p^{\text {put }}+p^{\text {call }}
$$

We do not have idiosyncratic risk in the model so the cross section of asset prices is degenerate. We also are not matching the VIX with the volatility of the price of capital. Instead, we are using the pricing kernel to price the put and call options. ${ }^{9}$

As before, we increase $\sigma$ and adjust the parameter pair $(\lambda, \theta)$ so that the long-run mean and variance of the growth rate do not change with their long-run values. We then simulate the model and calculate the price the security from equation (16), and price of options and VIX according to equations (17) to (19). The calculated VIX is further standardized to have zero mean and unit variance as in the empirical exercise.

Three series are plotted in Figure 8: the red line portrays data regression slope of GaR in the data on the VIX, while yellow and purple lines depict model-generated values under $15 \%$ and $30 \%$ increase in uncertainty $\tau$, respectively. It shows that an increase in the VIX decreases the GaR in the model for both values of $\sigma$. This is consistent with the feature in the data that higher asset price volatility magnifies the negative impact of uncertainty on IP growth. In addition, Figure 8 also shows that VIX has larger negative impact on $\mathrm{GaR}$ when there is higher uncertainty captured by $\sigma$. Compared to a naive empirical linear regression, our model produces a better fit for the relationship between VIX and GaR: it has $38 \%$ and $14 \%$ smaller root-mean squared error for $\sigma=1.05$ and

\footnotetext{
${ }^{9}$ It's worth noting that if the goal is to match stock market values using the price of firm capital, firm leverage should be taken into account (e.g. Bianchi, Ilut and Schneider (2017)).
} 


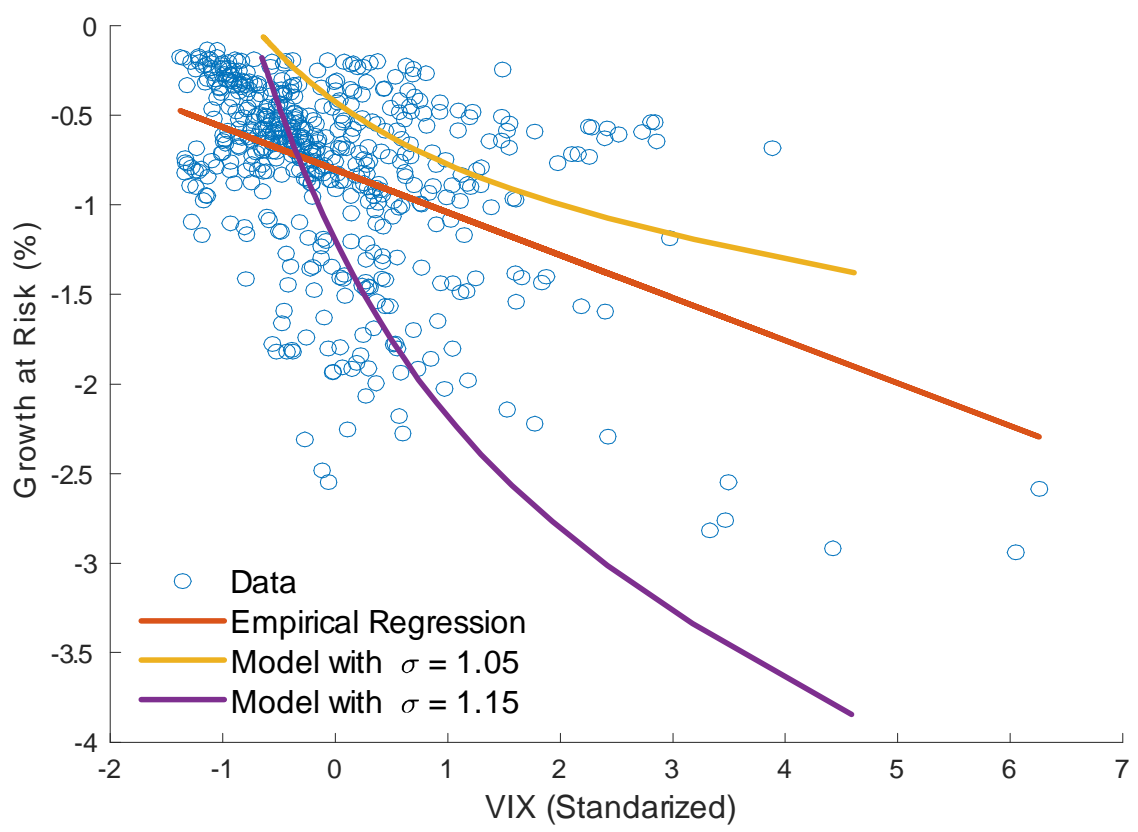

Figure 8: Asset Volatility and Growth-at-Risk. Note: The yellow (purple) reports modelgenerated contempraneous relationship between standardized asset volatility and Growth-at-Risk under $15 \%(30 \%)$ increase in uncertainty. The blue dots depict the empirical relationship between standardized VIX and Growth-at-Risk. The red line reports the OLS Estimate.

$\sigma=1.15$, respectively. ${ }^{10}$

\subsection{Capacity Utilization}

The model has no unemployed resources or spare capacity, but we may assume that the skill gap $u_{t}$ is an index of the fraction of capital - human or physical - that does not meet the needs of the date- $t$ technology. That interpretation of $u_{t}$ would match Ljungqvist and Sargent (1988) explanation for high European unemployment in the last two decades of the 20th century in which higher unemployment reflected restructuring from manufacturing to services, adoption of new information technologies, and globalization. And since Klein and $\mathrm{Su}$ (1979) a number of studies show a positive correlation between unemployment and capital utilization measures.

${ }^{10}$ The root-mean-squared error (RMSE) is defined as

$$
R M S E=\sqrt{\frac{1}{T} \sum_{i=1}^{T}\left(\hat{y}_{i}-y_{i}\right)^{2}}
$$

where the "hat" refers to the estimated value of GaR at time $t$. 
From equation (12), the detrended output in the model depends on the skill gap $u_{t}$. While it's empirically challenging to directly estimate the skill gap in the data, the capacity utilization rate - the ratio of actual output to the potential output - can be used to test the relationship between $u$ and the growth. The monthly data on U.S. capacity utilization is available at the FRED economic data maintained by the St. Louis Fed (series id TCU). As before, we simulate the model using calibrated parameters, and calculate skill gap $u_{t}$ according to equation (11). We define the capacity utilization as $1 /\left|u_{t}\right|$ so that an increase in the skill gap leads to a fall in capacity utilization. The calculated capacity utilization is further standardized to have zero mean and unit variance.

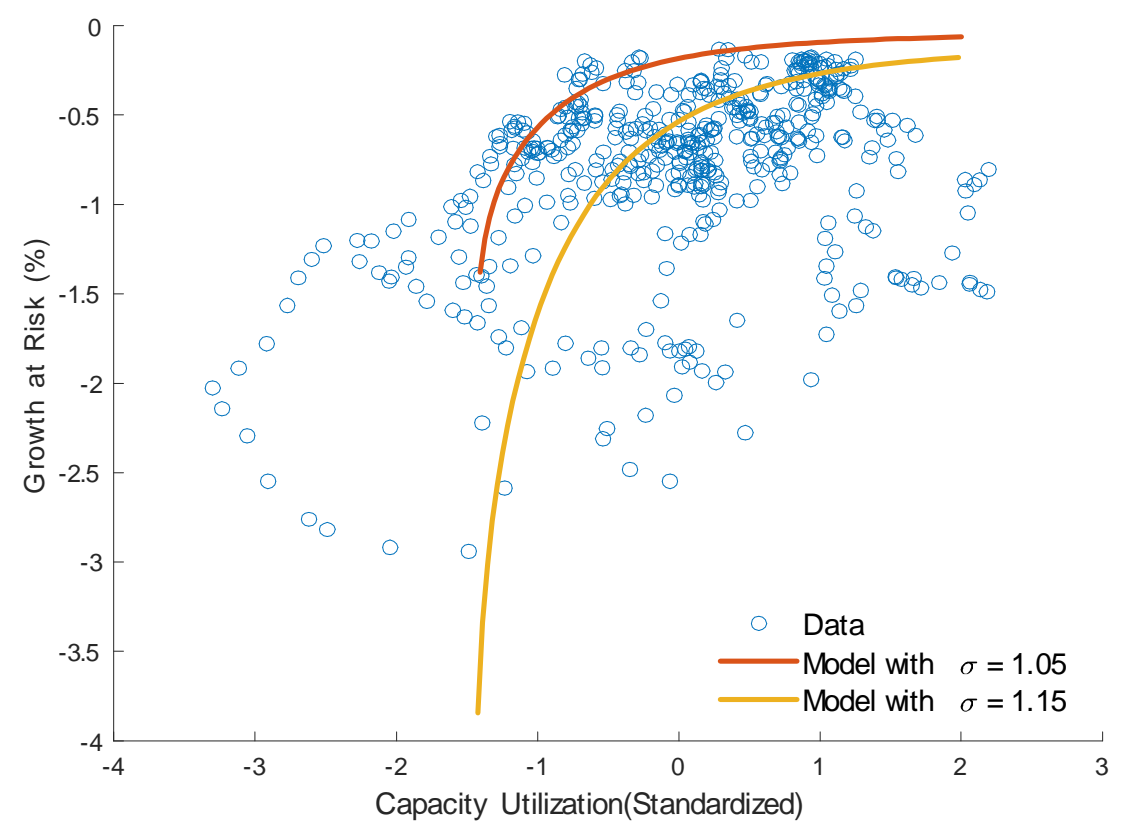

Figure 9: Capacity Utilization and Growth-at-Risk. Note: The red (yellow) reports modelgenerated contempraneous relationship between standardized capacity utilization and Growth-at-Risk under $15 \%$ (30\%) increase in uncertainty. The blue dots depict the empirical counterpart.

Figure 9 plots model implied Growth-at-Risk $\chi_{t}$ on the standardized capacity utilization for $\sigma=1.05$ and 1.15, respectively. It shows that, consistent with data, an decrease in the skill gap $u_{t}$ or an increase in capacity utilization increases the GaR. In addition, when the capacity utilization is high, an increase in uncertainty $(\sigma)$ has smaller negative impact on growth: a $10 \%$ increase $\sigma$ decreases $\mathrm{GaR}$ by $0.1 \%$ when capacity utilization is one standard deviation above its unconditional mean whereas decreases GaR by $0.9 \%$ when it is negative one standard deviation below its unconditional mean. 


\subsection{Long Run Growth Distribution}

Based on equation (14), growth distribution follows a negative chi-squared distribution in the long run. The top panel of Figure 10 depicts the simulated long run distribution for different values of $\sigma$. We again use calibrated $(\lambda, \theta)$ such that the mean and variance of the long run distribution remain the same. It shows higher uncertainty (higher $\sigma$ ) leads to a more negatively skewed distribution (portrayed by red bars) and lower 5th percentile (red dashed line).

The chi-squared distribution is well known as a light-tailed distribution whereas in the traditional growth literature (e.g. Barro and Jin (2011)), the heavy-tailed distribution, such as the Pareto Distribution, has been commonly used to calculate the tail risk. To address this issue, we assume that in the long horizon, the growth distribution follows,

$$
g_{t}=x-\psi x^{2} \sigma^{2} z_{t}
$$

The variable $z_{t} \in[0, \infty)$ follows a Type-II Pareto Distribution with associated CDF,

$$
F\left(z_{t}\right)=1-(1+x)^{-\alpha},
$$

where $\alpha$ is the shape parameter that governs the tail thickness.

We randomly draw $10,000 z_{t}$ from $F\left(z_{t}\right)$ and numerically calculate $\alpha$ by targeting the lower 5 th percentile to be the corresponding value in the calibrated Chi-squared distribution with $\sigma^{2}=1$. The bottom panel of Figure 10 depicts the simulated growth distribution using Pareto distribution with $\alpha=2.83$ (in red bars) against the baseline growth distribution with chi-squared distribution. It shows that Pareto distribution has a thicker tail than the Chi-squared distribution. Therefore, in order to generate the same downside risk, measured by the lower 5th percentile, the growth distribution with Pareto has much smaller mean growth $(0.78 \%)$ than the chi-squared distribution $(1.50 \%)$.

\subsection{Technological commitment}

It's worth noting that the assumption of irreversible technological commitment is essential to generate the negatively skewed growth distribution in equilibrium: output could fall because of commitment to technology before an unfavorable shock $\varepsilon$ is realized. If a firm could revert quickly and costlessly to technologies it used before, it would always use the best technology up to date and output never declines. For example, Jovanovic and Rob (1990) assume costless recall of past technologies. In contrast to our empirical evidence, instead of having a left tail, the distribution of growth rates in their model exhibits a spike at zero and a right tail. Therefore we need at least partial technological commitment. 

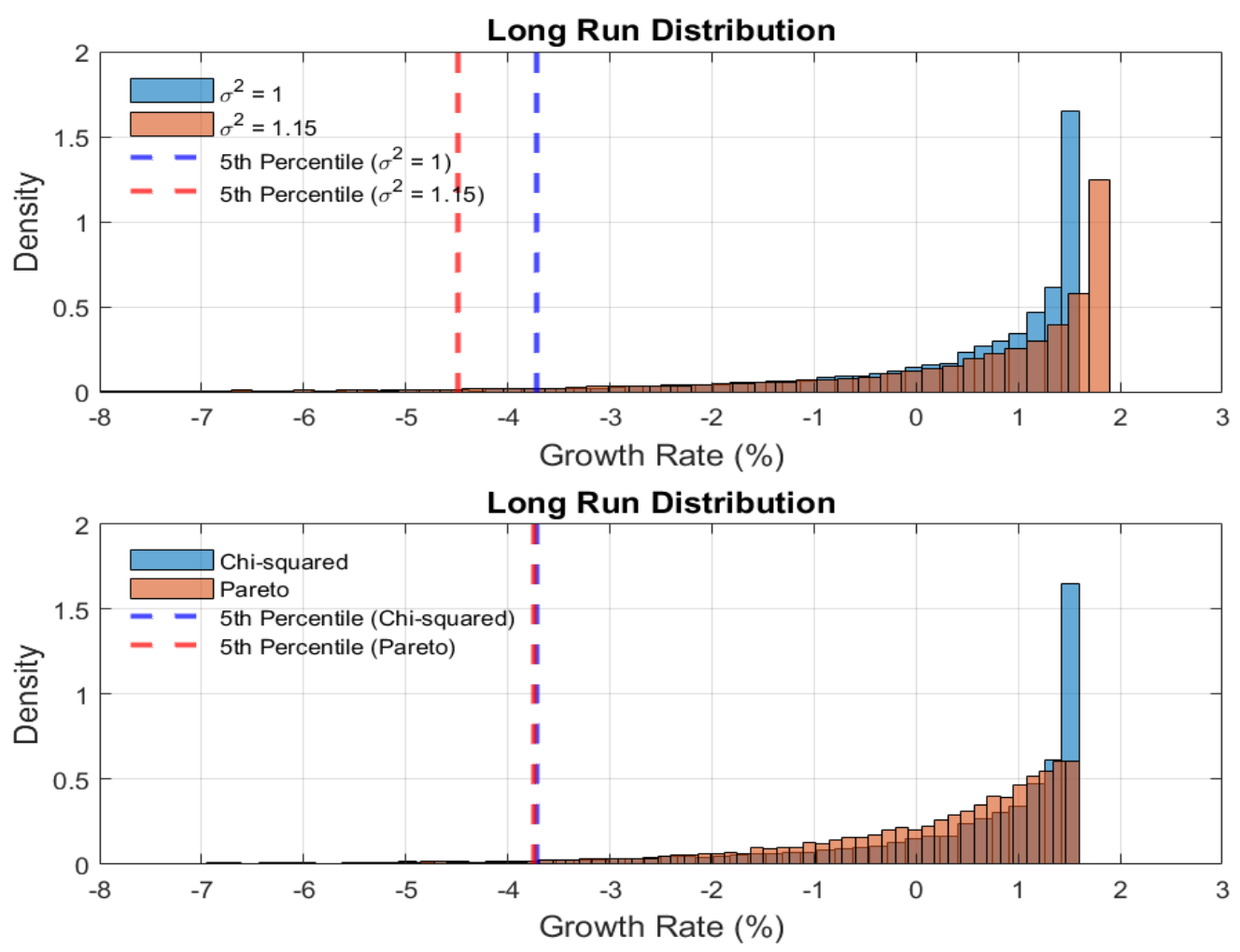

Figure 10: Simulated Long-run Growth Distribution. Note: the upper panel shows the simulated long-run growth distribution for small uncertainty (blue bars) and large uncertainty (red bars). The lower panel shows the simulated long-run growth distribution with Pareto distribution (red bars) and Chi-squared distribution (blue bars).

\subsection{Extension with Learning}

In the baseline model, we assume that the agent cannot directly learn the ideal skill. We relax this assumption in this section by adding the learning into the model and show that the qualitative result remains the same. As in the baseline model, the ideal skill $s$ is unobservable and the potential output follows,

$$
y^{p}=\exp \left\{A-\frac{\lambda}{2}(s-h)^{2}\right\}
$$

where for simplicity we assume that the ideal skill $s$ is the sum of two components

$$
s=\nu+\varepsilon_{t} .
$$

$\nu \in R$ is a parameter and $\varepsilon_{t}$ is i.i.d. with zero mean and variance of $\sigma_{\varepsilon}$. We further assume agent's prior over $\varepsilon_{t}$ is diffuse. 
Each period, the agent can purchase $n$ signals of parameter $\varepsilon_{t}$ with a cost (in foregone output),

$$
C\left(y^{p}, n\right) \equiv\left[1-\exp \left\{-\frac{\theta}{2} n^{2}\right\}\right] y^{p} .
$$

The net output therefore is

$$
y^{p}-C\left(y^{p}, n\right)=\exp \left\{A-\frac{\lambda}{2} u^{2}-\frac{\theta}{2} n^{2}\right\},
$$

where $u=s-h$ is the skill gap, and where $n$ is the number of independent signals on $\varepsilon_{t+1}$, call these signals, $\left(\xi_{1}, \ldots, \xi_{n, t}\right)$. Each $\xi_{i}$ is an independent signal on next period's $\varepsilon$ as follows:

$$
\xi_{i}=\varepsilon_{t+1}+\eta_{i}
$$

where $\eta_{i} \sim N\left(0, \sigma_{\eta}^{2}\right)$ are i.i.d. random variables. The agent forms the mean of the sample of $n$ signals:

$$
\bar{\xi}_{\mathrm{n}}=\frac{1}{n} \sum_{i=1}^{n} \xi_{i}=\varepsilon_{t+1}+\frac{1}{n} \sum_{i=1}^{n} \eta_{i} .
$$

Since each period $\varepsilon$ is independent of its past draws, $\bar{\xi}_{\mathrm{n}} \sim N\left(\varepsilon, \sigma_{\eta}^{2} / n\right)$ is a sufficient statistic for forecasting $\varepsilon$. And since the prior over $\varepsilon$ is diffuse, conditional on $\bar{\xi}_{\mathrm{n}}, \varepsilon \sim$ $N\left(\bar{\xi}_{\mathrm{n}}, \sigma_{\eta}^{2} / n\right)$. More formally,

$$
\lim _{\sigma_{\varepsilon} \rightarrow \infty} \operatorname{Pr}\left(\varepsilon \mid \bar{\xi}_{\mathrm{n}}\right)=\Phi\left(\frac{\varepsilon-\bar{\xi}_{\mathrm{n}}}{\sigma_{\eta}^{2} / n}\right),
$$

where $\Phi$ is the standard normal integral.

Denote $\tau$ the beginning-of-period prior variance over $\nu$, and $\tau^{\prime}$ the value next period. Then we have,

$$
\tau^{\prime}=\left(\frac{1}{\tau}+\frac{n}{\sigma_{\eta}^{2}}\right)^{-1} \equiv b(\tau, n),
$$

which shows the persisting benefits of $n$ via the reduction in $\tau$. As in Fajgelbaum et al. (2017), the notion of uncertainty is captured by the variance of beliefs $\tau$. Its law of motion is specified in equation (22).

In equilibrium, the agents set $h$ optimally to be the posterior mean and the next period's skill gap follows a normal distribution

$$
u^{\prime} \sim N\left(0, \tau+\frac{\sigma_{\eta}^{2}}{n}\right)
$$


or alternatively,

$$
u^{\prime}=\left(\tau+\frac{\sigma_{\eta}^{2}}{n}\right)^{1 / 2} \zeta
$$

where $\zeta$ follows a standardized normal distribution. Log output can be expressed as

$$
\ln y=A-\frac{\lambda}{2} u^{2}-\frac{\theta}{2} n^{2} .
$$

It's straightforward to verify that the state variable are $(u, \tau)$, which follow the law of motion,

$$
\begin{aligned}
u^{\prime} & =\left(\tau+\frac{\sigma_{\eta}^{2}}{n}\right)^{1 / 2} \zeta \\
\tau^{\prime} & =\left(\frac{1}{\tau}+\frac{n}{\sigma_{\eta}^{2}}\right)^{-1}
\end{aligned}
$$

The value function can be expressed as

$$
\begin{gathered}
V(\tau, u)=\max _{n} A-\frac{\lambda}{2} u^{2}-\frac{\theta}{2} n^{2}+\beta \int V\left(\left(\frac{1}{\tau}+\frac{n}{\sigma_{\eta}^{2}}\right)^{-1},\left(\tau+\frac{\sigma_{\eta}^{2}}{n}\right)^{1 / 2} \zeta\right) d F(\zeta) . \\
\text { s.t. } V(\tau, u) \geq 0
\end{gathered}
$$

Using equation (23), growth can be expressed as,

$$
\begin{aligned}
\Delta \ln y_{t+1} & =\frac{\lambda}{2}\left(u_{t}^{2}-u_{t+1}^{2}\right) \\
& =\frac{\lambda}{2}\left(u_{t}^{2}-\left(\tau+\frac{\sigma_{\eta}^{2}}{n}\right) \zeta^{2}\right) .
\end{aligned}
$$

First, we observe that the economy growth is driven by uncertainty though changes in the skill gap. Reducing the skill cap increases the growth and vice versa. Second, higher uncertainty leads to lower growth,

$$
\frac{\partial \Delta \ln y_{t+1}}{\partial \tau}=-\frac{\lambda}{2} \zeta^{2}<0
$$

Third, conditional on current skill gap $u_{t}$, the distribution of the growth is left skewed with the skewness,

$$
\operatorname{skewness}\left(\Delta \ln y_{t+1}\right)=-\lambda\left(\tau+\frac{\sigma_{\eta}^{2}}{n}\right) \Rightarrow \frac{\partial \text { skewness }\left(\Delta \ln y_{t+1}\right)}{\partial \tau}=-\lambda .
$$

Similar to the baseline model without learning, higher uncertainty leads to higher negative skewness and lower Growth-at-Risk. In the appendix, we provide an analytical solution to the distribution of growth in the equilibrium when the parameter $\nu$ is known. 


\section{Conclusion}

In this paper, we first empirically documented several stylized facts on the real effect of uncertainty. First, we showed that higher economic uncertainty is closely associated with a more dispersed and left-skewed growth distribution. Second, we found that the response of IP growth to an increase in uncertainty is highly nonlinear and asymmetric. Third, we presented evidence that higher asset volatility magnifies the negative impact of uncertainty on growth.

We presented and estimated a model in which rapid adoption of new technology may lead to higher economic uncertainty which causes measured productivity to decline. The model is able to match several key features in the data. The equilibrium growth distribution is negatively skewed and higher uncertainty leads to higher downside risk. The model is also able to generate a negative nonlinear relation between asset price volatility and Growth-at-Risk in equilibrium as observed in the data. 


\section{References}

Acemoglu, Daron and Andrew Scott, "Asymmetric business cycles: Theory and time-series evidence," Journal of Monetary Economics, 1997, 40 (3), 501-533.

Adrian, Tobias, Federico Grinberg, Nellie Liang, and Sheheryar Malik, The term structure of growth-at-risk, International Monetary Fund, 2018.

, Nina Boyarchenko, and Domenico Giannone, "Vulnerable growth," American Economic Review, 2019, 109 (4), 1263-89.

Alfaro, Ivan, Nicholas Bloom, and Xiaoji Lin, "The Real and Financial Impact of Uncertainty Shocks," in "Stanford Institute for Theoretical Economics (SITE) 2016 Workshop. Retrieved from https://site. stanford. edu/sites/default/files/alfaro. pdf" 2016.

Angelini, Giovanni, Emanuele Bacchiocchi, Giovanni Caggiano, and Luca Fanelli, "Uncertainty across volatility regimes," Journal of Applied Econometrics, 2019.

Arellano, Cristina, Yan Bai, and Patrick Kehoe, "Financial Markets and Fluctuations in Uncertainty," January 2011. Federal Reserve Bank of Minneapolis Research Department Staff Report 466.

Baker, Scott R, Nicholas Bloom, and Steven J Davis, "Measuring economic policy uncertainty," The Quarterly Journal of Economics, 2016, 131 (4), 1593-1636.

Barro, Robert J and Tao Jin, "On the size distribution of macroeconomic disasters," Econometrica, 2011, 79 (5), 1567-1589.

Basu, Susanto and Brent Bundick, "Uncertainty shocks in a model of effective demand," Econometrica, 2017, 85 (3), 937-958.

Bernanke, Ben S., "Irreversibility, Uncertainty, and Cyclical Investment," The Quarterly Journal of Economics, 1983, 98 (1), 85-106.

Bianchi, Francesco, Cosmin L Ilut, and Martin Schneider, "Uncertainty shocks, asset supply and pricing over the business cycle," The Review of Economic Studies, $2017,85(2), 810-854$.

Bloom, Nicholas, "The Impact of Uncertainty Shocks," Econometrica, May 2009, 77 (3), 623-85.

, Max Floetotto, Nir Jaimovich, Itay Saporta-Eksten, and Stephen J Terry, "Really uncertain business cycles," Econometrica, 2018, 86 (3), 1031-1065. 
Caldara, Dario, Cristina Fuentes-Albero, Simon Gilchrist, and Egon Zakrajšek, "The macroeconomic impact of financial and uncertainty shocks," European Economic Review, 2016, 88, 185-207.

Campbell, Jeffrey R, "Entry, exit, embodied technology, and business cycles," Review of economic dynamics, 1998, 1 (2), 371-408.

Carriero, Andrea, Todd E Clark, and Massimiliano Marcellino, "Measuring uncertainty and its impact on the economy," Review of Economics and Statistics, 2018, $100(5), 799-815$.

Chalkley, Martin and In Ho Lee, "Learning and asymmetric business cycles," Review of Economic Dynamics, 1998, 1 (3), 623-645.

Fajgelbaum, Pablo D, Edouard Schaal, and Mathieu Taschereau-Dumouchel, "Uncertainty traps," The Quarterly Journal of Economics, 2017, 132 (4), 1641-1692.

Fernández-Villaverde, Jesús, Juan F. Rubio-Ramírez Pablo GuerrónQuintana, and Martin Uribe, "Risk Matters: The Real Effects of Volatility Shocks," American Economic Review, October 2011, 6 (101), 2530-61.

Gilchrist, Simon and John C Williams, "Putty-clay and investment: a business cycle analysis," Journal of Political Economy, 2000, 108 (5), 928-960.

, Jae W. Sim, and Egon Zakrajsek, "Uncertainty, Financial Frictions, and Investment Dynamics," June 2010. Society for Economic Dynamics 2010 Meeting Papers No.1285.

Hengge, Martina, "Uncertainty as a Predictor of Economic Activity," 2019.

Johansen, Leif, "Substitution versus fixed production coefficients in the theory of economic growth: a synthesis," Econometrica: Journal of the Econometric Society, 1959, pp. $157-176$.

Jovanovic, Boyan, "Asymmetric cycles," The Review of Economic Studies, 2006, 73 (1), 145-162.

and Rafael Rob, "Long waves and short waves: Growth through intensive and extensive search," Econometrica: Journal of the Econometric Society, 1990, pp. 13911409 .

Jurado, Kyle, Sydney C. Ludvigson, and Serena Ng, "Measuring Uncertainty," The American Economic Review, 2015, 105 (3), 117-1216.

Klein, Lawrence $\mathbf{R}$ and Vincent $\mathbf{S u}$, "Direct estimates of unemployment rate and capacity utilization in macroeconometric models," International Economic Review, 1979, pp. $725-740$. 
Klenow, Peter J, "Learning curves and the cyclical behavior of manufacturing industries," Review of Economic dynamics, 1998, 1 (2), 531-550.

Leduc, Sylvain and Zheng Liu, "Uncertainty shocks are aggregate demand shocks," Journal of Monetary Economics, 2016, 82, 20-35.

Ljungqvist, L and T Sargent, "The European Unemployment Dilemma," , Journal of Political Economy, 1988.

Lucas, Robert, "Asset Prices in an Exchange Economy," Econometrica, 1978, 46, 14291446.

Ludvigson, Sydney C., Sai $\mathrm{Ma}$, and Serena $\mathbf{N g}$, "Uncertainty and Business Cycles: Exogenous Impulse or Endogenous Response?," American Economic Journal: Macroeconomics, forthcoming, 2019. http://www.econ.nyu.edu/user/ludvigsons/ucc.pdf.

McCracken, Michael W and Serena Ng, "FRED-MD: A monthly database for macroeconomic research," Journal of Business \& Economic Statistics, 2016, 34 (4), 574589.

McDonald, Robert and Daniel Siegel, "The Value of Waiting to Invest," The Quarterly Journal of Economics, 1986, 101 (4), 707-728.

Prescott, Edward C and Michael Visscher, "Organization capital," Journal of political Economy, 1980, 88 (3), 446-461.

Ramey, Garey and Valerie A Ramey, "Technology commitment and the cost of economic fluctuations," Technical Report, National Bureau of Economic Research 1991.

Shin, Minchul and Molin Zhong, "A new approach to identifying the real effects of uncertainty shocks," Journal of Business 8 Economic Statistics, 2018, pp. 1-13.

Veldkamp, Laura L, "Slow boom, sudden crash," Journal of Economic theory, 2005, $124(2), 230-257$. 


\section{Appendix}

\subsection{Proof of Proposition 1}

The first-order condition for the optimality of $\Delta$ is

$$
\lambda(u-\Delta)-\theta \Delta-\beta \int V_{1} d F=0
$$

and the first-order condition for the optimality of $x$ is

$$
\int\left(\varepsilon V_{1}+V_{2}\right) d F=0
$$

Solving for $\Delta$.- The envelope theorem gives

$$
V_{1}=-\lambda(u-\Delta)+\beta \int V_{1} d F=-\theta \Delta,
$$

where the second equality uses (24). Substituting into (24) we have

$$
\lambda(u-\Delta)=\theta \Delta-\beta \theta \int \Delta^{\prime} d F(\varepsilon) .
$$

We seek a solution of the form (7) where $\alpha$ is a constant to be solved for. If (7) holds, (27) reads

$$
\begin{aligned}
\lambda \alpha u & =\theta(1-\alpha) u-\beta \theta \int(1-\alpha)(\alpha u+x \varepsilon) d F(\varepsilon) \\
& =\theta(1-\alpha) u-\beta \theta(1-\alpha) \alpha u
\end{aligned}
$$

which, after cancellation of $u$ leaves a quadratic in $\alpha$, namely $\theta(1-\alpha)-\beta \theta(1-\alpha) \alpha-$ $\lambda \alpha=0$, or

$$
\beta \alpha^{2}-\left(1+\beta+\frac{\lambda}{\theta}\right) \alpha+1=0
$$

This implicit function has the solution for $\alpha$ given in (8).

Solving for $x$.- The envelope theorem also gives

$$
V_{2}=1+\beta \int V_{2} d F=\frac{1}{1-\beta} .
$$

The second equality follows because the right-hand side of (29) is a contraction map with at most one solution for $V_{2}$. Substituting from (7) into (26) and from there (in an updated form) into (25) gives

$$
\begin{aligned}
0 & =-\int \varepsilon \theta \Delta^{\prime} d F+\frac{1}{1-\beta} \\
& =-\int \varepsilon \theta(1-\alpha)(\alpha u+x \varepsilon) d F+\frac{1}{1-\beta}
\end{aligned}
$$


because $\Delta^{\prime}=(1-\alpha) u^{\prime}=(1-\alpha)(\alpha u+x \varepsilon)$. But $E(\varepsilon u)=0$, which leads to $(6)$.

We must show that this function solves (5). Let us proceed with the method of undetermined coefficients. Since $V=a A-b u^{2}+c$,

$$
\begin{aligned}
a A-b u^{2}+c & =A-\psi u^{2}+\beta \int\left(a(A+x)-b(u+x \varepsilon-\Delta)^{2}+c\right) d F(\varepsilon) \\
& =A-\psi u^{2}+\beta \int\left(a(A+x)-b(\alpha u+x \varepsilon)^{2}+c\right) d F(\varepsilon) \\
& =A-\psi u^{2}+\beta(a(A+x)+c)-\beta b \int(\alpha u+x \varepsilon)^{2} d F(\varepsilon) \\
& =A-\psi u^{2}+\beta(a(A+x)+c)-\beta b \alpha^{2} u^{2}-\beta b x^{2} \sigma^{2}
\end{aligned}
$$

Equating coefficients: $a=1+a \beta, b=\psi+b \beta \alpha^{2}$, and $c=\beta\left(a x+c-b x^{2} \sigma^{2}\right)$, so that

$$
a=\frac{1}{1-\beta}, \quad b=\frac{\psi}{1-\beta \alpha^{2}}, \text { and } c=\frac{\beta}{1-\beta}\left(a x-b x^{2} \sigma^{2}\right) .
$$

This leads to

$$
V(u, A)=\frac{A}{1-\beta}-\frac{\psi}{1-\beta \alpha^{2}} u^{2}+\frac{\beta\left(\frac{x}{1-\beta}-\left[\frac{\psi}{1-\beta \alpha^{2}}\right] x^{2} \sigma^{2}\right)}{1-\beta},
$$

where $x=\frac{1}{\theta \sigma^{2}(1-\beta)(1-\alpha)}$. Then $V_{2}(u, A)=1 /(1-\beta)$ which is consistent with (29). It remains to be shown that $V_{1}(u, A)$ agrees with (26) and (7). Now, since $\psi=\frac{1}{2}\left(\lambda \alpha^{2}+\theta(1-\alpha)^{2}\right)$, they agree only if

$$
\frac{\psi}{1-\beta \alpha^{2}}=\frac{1}{2} \theta(1-\alpha)
$$

i.e., if

$$
\left(\frac{\lambda}{\theta} \alpha^{2}+(1-\alpha)^{2}\right)=(1-\alpha)\left(1-\beta \alpha^{2}\right)
$$

But from (28),

$$
\frac{\lambda}{\theta}=\frac{1}{\alpha}+\beta \alpha-1-\beta
$$

Substitute for $\lambda / \theta$ into (31) to conclude that $V_{1}(u, A)$ is consistent with (26) and (7) if and only if

$$
\left(\alpha+\beta \alpha^{3}-\alpha^{2}-\beta \alpha^{2}+(1-\alpha)^{2}\right)=(1-\alpha)\left(1-\beta \alpha^{2}\right)
$$

But expanding the left-hand side of (32) yields

$$
\left(\alpha+\beta \alpha^{3}-\alpha^{2}-\beta \alpha^{2}+1+\alpha^{2}-2 \alpha\right)=\beta \alpha^{3}-\beta \alpha^{2}+1-\alpha .
$$

Conversely, expanding the right-hand side of (32) yields

$$
(1-\alpha)\left(1-\beta \alpha^{2}\right)=1-\alpha-\beta \alpha^{2}+\beta \alpha^{3} .
$$

Therefore (32) holds, and $V$ is therefore given by (9). 


\subsection{Proof of Proposition 2}

Note that we can re-write 11 as $M A(t)$ process

$$
u_{t}=\alpha^{t} u_{0}+x \sum_{s=0}^{t} \alpha^{t-s} \varepsilon_{s} .
$$

As a result, we have

$$
\begin{aligned}
u_{t+1}^{2}-u_{t}^{2}= & \left(u_{t+1}+u_{t}\right)\left(u_{t+1}-u_{t}\right) \\
= & \left\{\left(\alpha^{t+1}+\alpha^{t}\right) u_{0}+x\left\{\sum_{s=0}^{t+1} \alpha^{t+1-s} \varepsilon_{s}+\sum_{s=0}^{t} \alpha^{t-s} \varepsilon_{s}\right\}\right\} \\
& \left\{\left(\alpha^{t+1}-\alpha^{t}\right) u_{0}+x\left\{\sum_{s=0}^{t+1} \alpha^{t+1-s} \varepsilon_{s}-\sum_{s=0}^{t} \alpha^{t-s} \varepsilon_{s}\right\}\right\} \\
= & \{\underbrace{\left\{\left(\alpha^{t+1}+\alpha^{t}\right) u_{0}+x\left\{2 \sum_{s=0}^{t} \alpha^{t-s} \varepsilon_{s}+\varepsilon_{t+1}\right\}\right\}}_{\zeta_{1}} \underbrace{\left\{\left(\alpha^{t+1}-\alpha^{t}\right) u_{0}+x \varepsilon_{t+1}\right\}}_{\zeta_{2}} .
\end{aligned}
$$

This is product of two variables $\zeta_{1}$ and $\zeta_{2}$ that follow normal distributions

$$
\begin{aligned}
& \zeta_{1} \sim N\left(\left(\alpha^{t+1}+\alpha^{t}\right) u_{0}, x^{2}\left\{4\left[\frac{1-\alpha^{2(t+1)}}{1-\alpha^{2}}\right]+1\right\} \sigma^{2}\right) \\
& \zeta_{2} \sim N\left(\left(\alpha^{t+1}-\alpha^{t}\right) u_{0}, x^{2} \sigma^{2}\right) .
\end{aligned}
$$

It's worth noting that the product of two variables can be written as

$$
X Y=\frac{1}{4}(X+Y)^{2}-\frac{1}{4}(X-Y)^{2}
$$

It follows that since $X=\zeta_{1}$ and $Y=\zeta_{2}$ are normal distribution

$$
\begin{aligned}
\zeta_{1}+\zeta_{2}-2 \alpha^{t+1} u_{0} & \sim N\left(0,4 x^{2}\left[\left[\frac{1-\alpha^{2(t+1)}}{1-\alpha^{2}}\right]+1\right] \sigma^{2}\right)=A \xi_{t} \\
\zeta_{1}+\zeta_{2}-2 \alpha^{t} u_{0} & \sim N\left(0,4 x^{2}\left[\frac{1-\alpha^{2(t+1)}}{1-\alpha^{2}}\right] \sigma^{2}\right)=B \xi_{t} .
\end{aligned}
$$

where $\xi_{t}$ is a standard normal and

$$
\begin{aligned}
A & =\sqrt{4 x^{2} \sigma^{2}\left[\left[\frac{1-\alpha^{2(t+1)}}{1-\alpha^{2}}\right]+1\right]} \\
B & =\sqrt{4 x^{2}\left[\frac{1-\alpha^{2(t+1)}}{1-\alpha^{2}}\right] \sigma^{2} .}
\end{aligned}
$$


Therefore, we have

$$
\begin{aligned}
\zeta_{1} \zeta_{2}= & \frac{1}{4}\left(A \xi_{t}+2 \alpha^{t+1} u_{0}\right)^{2}-\frac{1}{4}\left(B \xi_{t}+2 \alpha^{t} u_{0}\right)^{2} \\
= & \frac{1}{4}\left(\left[A \xi_{t}\right]^{2}+\left[2 \alpha^{t+1} u_{0}\right]^{2}+4 \alpha^{t+1} u_{0} A \xi_{t}\right) \\
& -\frac{1}{4}\left(\left[B \xi_{t}\right]^{2}+\left[2 \alpha^{t} u_{0}\right]^{2}+4 \alpha^{t} u_{0} B \xi_{t}\right) \\
= & C+\frac{1}{4}\left[A^{2}-B^{2}\right] \xi_{t}^{2}+(\alpha A-B) \alpha^{t} u_{0} \xi_{t}
\end{aligned}
$$

where the constant

$$
C=\left(\alpha^{t+1} u_{0}\right)^{2}-\left(\alpha^{t} u_{0}\right)^{2}
$$

Now note that

$$
A^{2}-B^{2}=4 x^{2} \sigma^{2}
$$

this leads to

$$
\zeta_{1} \zeta_{2}=C+x^{2} \sigma^{2} \xi_{t}^{2}+(\alpha A-B) \alpha^{t} u_{0} \xi_{t}
$$

We now have the growth distribution

$$
g_{t}=x-\psi\left[C_{t}+x^{2} \sigma^{2} \xi_{t}^{2}+(\alpha A-B) \alpha^{t} u_{0} \xi_{t}\right]
$$

where $\xi_{t} \sim N(0,1)$ and $A, B$ and $C$ are as stated in the proposition.

\subsection{Extension with Learning}

The following proposition provides a closed-form expression for the growth distribution when there is no uncertainty of $\nu$.

Proposition 3 When there is no uncertainty of $\nu$ and under log preferences, the optimal policy function and value function satisfy,

$$
\begin{aligned}
\Delta & =\left(\frac{\psi \beta \sigma^{2}}{\theta}\right)^{\frac{1}{3}} \\
V(u) & =B-\psi u^{2},
\end{aligned}
$$

where

$$
\begin{aligned}
B & =\frac{1}{1-\beta}\left(A-\frac{\theta}{2}\left(\frac{\psi \beta \sigma^{2}}{\theta}\right)^{\frac{2}{3}}-\psi \beta \sigma^{2}\left(\frac{\psi \beta \sigma^{2}}{\theta}\right)^{-\frac{1}{3}}\right) \\
\psi & =\frac{\lambda^{2}}{2}
\end{aligned}
$$


and the log growth $g$ follows

$$
g=\Delta \ln y=\frac{\lambda}{2}\left(u^{2}-\left(\frac{\psi \beta \sigma^{2}}{\theta}\right)^{-\frac{1}{3}} \sigma^{2} \zeta^{2}\right) .
$$

Therefore conditional on $u$, the growth distribution is left skewed

$$
\begin{aligned}
E(g) & =\frac{\lambda}{2} u^{2}-\frac{\lambda \sigma^{2}}{2}\left(\frac{\psi \beta \sigma^{2}}{\theta}\right)^{-\frac{1}{3}} \\
\operatorname{var}(g) & =2\left(\frac{\lambda \sigma^{2}}{2}\left(\frac{\psi \beta \sigma^{2}}{\theta}\right)^{-\frac{1}{3}} \frac{\sigma^{2}}{\Delta}\right)^{2} \\
\text { skewness }(g) & =-\frac{\lambda \sigma^{2} \sqrt{8}}{2}\left(\frac{\psi \beta \sigma^{2}}{\theta}\right)^{-\frac{1}{3}} .
\end{aligned}
$$

Proof. When there is no uncertainty of $\nu, \tau=0$,

$$
V(u)=\max _{\Delta} A-\frac{\lambda}{2} u^{2}-\frac{\theta}{2} \Delta^{2}+\beta \int V\left(\left(\frac{\sigma^{2}}{\Delta}\right)^{1 / 2} \zeta\right) d F(\zeta)
$$

If we guess and verify $V(u)=B-\psi u^{2}$ and $\Delta=C$

$$
\begin{aligned}
V(u) & =\max _{\Delta} A-\frac{\lambda}{2} u^{2}-\frac{\theta}{2} \Delta^{2}+\beta \int V\left(\left(\frac{\sigma^{2}}{\Delta}\right)^{1 / 2} \zeta\right) d F(\zeta) \\
& =\max _{\Delta} A-\frac{\lambda}{2} u^{2}-\frac{\theta}{2} \Delta^{2}+\beta B-\psi \beta\left(\frac{\sigma^{2}}{\Delta}\right)
\end{aligned}
$$

First order condition

$$
\psi \beta\left(\frac{\sigma^{2}}{\Delta^{2}}\right)=\theta \Delta
$$

or

$$
\Delta=\left(\frac{\psi \beta \sigma^{2}}{\theta}\right)^{\frac{1}{3}}
$$

For the value function,

$$
B-\psi u^{2}=A-\frac{\lambda}{2} u^{2}-\frac{\theta}{2} \Delta^{2}+\beta B-\psi \beta\left(\frac{\sigma^{2}}{\Delta}\right)
$$

Matching the coefficients,

or

$$
B=A-\frac{\theta}{2} \Delta^{2}+\beta B-\psi \beta\left(\frac{\sigma^{2}}{\Delta}\right)
$$

$$
\begin{aligned}
B & =\frac{1}{1-\beta}\left(A-\frac{\theta}{2}\left(\frac{\psi \beta \sigma^{2}}{\theta}\right)^{\frac{2}{3}}-\psi \beta \sigma^{2}\left(\frac{\psi \beta \sigma^{2}}{\theta}\right)^{-\frac{1}{3}}\right) \\
\psi & =\frac{\lambda}{2},
\end{aligned}
$$

as stated in the proposition. 